\title{
Integrable pseudopotentials related to generalized hypergeometric functions
}

\author{
A.V. Odesskii ${ }^{1,2}$, V.V. Sokolov ${ }^{1}$ \\ ${ }^{1}$ L.D. Landau Institute for Theoretical Physics (Russia) \\ ${ }^{2}$ University of Manchester (UK)
}

\begin{abstract}
We construct integrable pseudopotentials with an arbitrary number of fields in terms of generalized hypergeometric functions. These pseudopotentials yield some integrable $(2+1)$-dimensional hydrodynamic type systems. In two particular cases these systems are equivalent to integrable scalar 3dimensional equations of second order. An interesting class of integrable $(1+1)$-dimensional hydrodynamic type systems is also generated by our pseudopotentials.
\end{abstract}

MSC numbers: 17B80, 17B63, 32L81, 14H70

Address: L.D. Landau Institute for Theoretical Physics of Russian Academy of Sciences, Kosygina 2, 119334, Moscow, Russia

E-mail: alexander.odesskii@manchester.ac.uk, odesskii@itp.ac.ru, sokolov@itp.ac.ru 


\section{Contents}

1 Introduction

2 Generalized hypergeometric functions

3 Pseudopotentials of defect 0

4 Pseudopotentials of defect $k>0$

11

5 Integrable $(1+1)$-dimensional systems of hydrodynamic type

6 Hydrodynamic reductions and integrability

7 Conclusion

25 


\section{Introduction}

The main object of integrability theory is the Lax equation

$$
L_{t}=[L, A]
$$

Here $A$ and $L$ are operators depending on functions $u_{1}, \ldots, u_{n}$ and (1.1) is equivalent to a system of nonlinear differential equations for $u_{i}$. For the KP-hierarchy and its different reductions $A$ is a linear differential operator $A=\sum r_{i} \partial_{x}^{i}$, whose coefficients $r_{i}$ are differential polynomials in $u_{1}, \ldots, u_{n}$. The $L$-operator could be a differential operator or a more complicated object like a ratio of two differential operators or a formal (non-commutative) Laurent series with respect to $\partial_{x}^{-1}$.

The dispersionless analog of (1.1) has the following form

$$
L_{t}=\{L, A\}
$$

where $\{L, A\}=A_{p} L_{x}-A_{x} L_{p}$. As usual, the commutator in (1.1) is replaced by the Poisson bracket and the non-commutative variable $\partial_{x}$ by the commutative "spectral" parameter $p$. The transformation $L(x, t, p) \rightarrow p(x, t, L)$ reduces (1.2) to the following conservative form

$$
p_{t}=A\left(p, u_{1}, \ldots, u_{n}\right)_{x}
$$

where $L$ plays the role of a parameter. The latter equation can be rewritten as

$$
\psi_{t}=A\left(\psi_{x}, u_{1}, \ldots, u_{n}\right)
$$

where $p=\psi_{x}$.

Equations (1.4) can be chosen as a basis, on which a theory of integrable 3-dimensional dispersionless PDEs can be built. Most such equations can be written in the form

$$
\sum_{j=1}^{n} a_{i j}(\mathbf{u}) u_{j, t_{1}}+\sum_{j=1}^{n} b_{i j}(\mathbf{u}) u_{j, t_{2}}+\sum_{j=1}^{n} c_{i j}(\mathbf{u}) u_{j, t_{3}}=0, \quad i=1, \ldots, l,
$$

where $\mathbf{u}=\left(u_{1}, \ldots, u_{n}\right)$. All known integrable systems (1.5) admit the so-called pseudopotential representation

$$
\psi_{t_{2}}=A\left(\psi_{t_{1}}, \mathbf{u}\right), \quad \psi_{t_{3}}=B\left(\psi_{t_{1}}, \mathbf{u}\right),
$$

by means of a pair of equations (1.4) whose the compatibility conditions $\psi_{t_{2} t_{3}}=\psi_{t_{3} t_{2}}$ are equivalent to (1.5). The functions $A, B$ are called pseudopotentials. Such a pseudopotential representation is a dispersionless version of the zero curvature representation, which is a basic notion in the integrability theory of solitonic equations (see [1]) .

One of the interesting and attractive features of the theory of integrable dispersionless equations is that the dependence of the pseudopotentials $A\left(p, u_{1}, \ldots, u_{n}\right)$ on $p$ can be much more 
complicated then in the solitonic case. For instance, in [2, 3] some important examples of pseudopotentials $A$ were found related to the Whitham averaging procedure for integrable dispersion PDEs and to the Frobenious manifolds. For these examples the $p$-dependence is determined by an algebraic curve of arbitrary genus $g$. In the paper [4] a certain class of pseudopotentials with movable singularities was described. Some of the pseudopotentials constructed in [4] are written in terms of degenerate hypergeometric functions.

In the paper [5] a wide class of pseudopotentials $A\left(p, u_{1}, \ldots, u_{n}\right)$ related to rational algebraic curves was constructed. These pseudopotentials were written in the following parametric form:

$$
A=F_{1}\left(\xi, u_{1}, \ldots, u_{n}\right), \quad p=F_{2}\left(\xi, u_{1}, \ldots, u_{n}\right),
$$

where the $\xi$-dependence of the functions $F_{i}$ is defined by the ODE

$$
F_{i, \xi}=\phi_{i}\left(\xi, u_{1}, \ldots, u_{n}\right) \cdot \xi^{-s_{1}}(\xi-1)^{-s_{2}}\left(\xi-u_{1}\right)^{-s_{3}} \ldots\left(\xi-u_{n}\right)^{-s_{n+2}} .
$$

Here $s_{1}, \ldots, s_{n+2}$ are arbitrary constants and $\phi_{i}$ are polynomials in $\xi$ of degree $n$. The dependence of $\phi_{i}$ on $u_{1}, \ldots, u_{n}$ was described in terms of solutions of some overdetermined linear system of PDEs with rational coefficients.

In this paper we generalize this result and construct new classes of pseudopotentials $A_{n, k}\left(p, u_{1}, \ldots, u_{n}\right)$ whose $p$-dependence is given by (1.7), where $\phi_{i}(\xi)$ are polynomials in $\xi$ of degree $n-k, k=0, \ldots, n-1$. We call the corresponding functions $A_{n, k}$ pseudopotentials of defect $k$. The pseudopotentials of defect 0 are just pseudopotentials from [5] written in a different form.

We describe the pseudopotentials of defect $k$ in terms of linearly independent solutions of the following system of linear PDEs with rational coefficients

$$
\frac{\partial^{2} h}{\partial u_{i} \partial u_{j}}=\frac{s_{i}}{u_{i}-u_{j}} \cdot \frac{\partial h}{\partial u_{j}}+\frac{s_{j}}{u_{j}-u_{i}} \cdot \frac{\partial h}{\partial u_{i}}, \quad i, j=1, \ldots, n, \quad i \neq j,
$$

and

$$
\begin{aligned}
\frac{\partial^{2} h}{\partial u_{i} \partial u_{i}}=-(1+ & \left.\sum_{j=1}^{n+2} s_{j}\right) \frac{s_{i}}{u_{i}\left(u_{i}-1\right)} h+\frac{s_{i}}{u_{i}\left(u_{i}-1\right)} \sum_{j \neq i}^{n} \frac{u_{j}\left(u_{j}-1\right)}{u_{j}-u_{i}} \cdot \frac{\partial h}{\partial u_{j}}+ \\
& \left(\sum_{j \neq i}^{n} \frac{s_{j}}{u_{i}-u_{j}}+\frac{s_{i}+s_{n+1}}{u_{i}}+\frac{s_{i}+s_{n+2}}{u_{i}-1}\right) \frac{\partial h}{\partial u_{i}}
\end{aligned}
$$

for one unknown function $h\left(u_{1}, \ldots, u_{n}\right)$. If $n=1$, then we have no equations (1.8) and the single equation (1.9) coincides with the standard hypergeometric equation,

$$
u(u-1) h(u)^{\prime \prime}+[(\alpha+\beta+1) u-\gamma] h(u)^{\prime}+\alpha \beta h(u)=0,
$$

where $s_{1}=-\alpha, s_{2}=\alpha-\gamma, s_{3}=\gamma-\beta-1$. Notice, that hypergeometric functions already appeared in connection with dispersionless PDEs (see, for example [6, 4, 7]). For arbitrary 
$n$ the system (1.8), (1.9) can be solved in terms of generalized hypergeometric functions (see [8, 9]).

Note that the pseudopotential $A_{n, k}$ is written in terms of $k+2$ linearly independent solutions of the system (1.8), (1.9) and therefore the group $G L_{k+2}$ acts on the set of such pseudopotentials. If $k=0$, then this is just the usual action of $G L_{2}$ on the space of independent variables $x, t$ in the equation (1.4). In the case $k>0$ the action of larger group $G L_{k+2}$ is still to be explained. For a particular class of 3-dimensional equations the existence of such a group of symmetries was pointed out in [10] (see also [11]). It is known [12, 10] that knowledge of the symmetry group $G L_{n}$ allows us to linearize systems of ODEs and PDEs.

The paper is organized as follows.

In Section 2 we describe some properties of system (1.8), (1.9) and its solutions needed for our purposes. Most of these properties are well known to experts.

In Section 3 we rewrite formulas of the paper [5] in terms of generalized hypergeometric functions. In our paper the pseudopotentials constructed in [5] are called pseudopotentials of defect 0 . A couple of such pseudopotentials defines a system of the form (1.5) with $l=n$. These systems are rewritten in terms of generalized hypergeometric functions in Section 3 . We also prove that each of these systems admits $n+1$ conservation laws of hydrodynamic type.

In Section 4, for any $n$ and $k>0$ we construct pseudopotentials of defect $k$. A couple of such pseudopotentials defines a system of the form (1.5) with $l=n+k$. These systems are also constructed in Section 4. The particular cases $n=3, k=1$ and $n=5, k=3$ yield integrable equations of the form

$$
\sum_{i, j} P_{i, j}\left(z_{t_{1}}, z_{t_{2}}, z_{t_{3}}\right) z_{t_{i}, t_{j}}=0, \quad i, j=1,2,3
$$

and

$$
Q\left(z_{t_{1}, t_{1}}, z_{t_{1}, t_{2}}, z_{t_{1}, t_{3}}, z_{t_{2}, t_{2}}, z_{t_{2}, t_{3}}, z_{t_{3}, t_{3}}\right)=0 .
$$

A classification of all integrable equations (1.10) and (1.11) was presented in [1] and in [13], correspondingly. Our integrable equations give generic solutions of these classification problems.

In Sections 5 we construct and study a certain class of integrable $(1+1)$-dimensional hydrodynamic type systems of the form

$$
r_{t}^{i}=v^{i}\left(r^{1}, \ldots, r^{N}\right) r_{x}^{i}, \quad i=1,2, \ldots, N .
$$

These systems are defined by an universal overdetermined compatible system of PDEs of the Gibbons-Tsarev type [14, 15] for some functions $w\left(r^{1}, \ldots, r^{N}\right), \xi_{1}\left(r^{1}, \ldots, r^{N}\right), \ldots, \xi_{N}\left(r^{1}, \ldots, r^{N}\right)$. This system has the following form

$$
\partial_{i} \xi_{j}=\frac{\xi_{j}\left(\xi_{j}-1\right)}{\xi_{i}-\xi_{j}} \partial_{i} w, \quad \partial_{i j} w=\frac{2 \xi_{i} \xi_{j}-\xi_{i}-\xi_{j}}{\left(\xi_{i}-\xi_{j}\right)^{2}} \partial_{i} w \partial_{j} w, \quad i, j=1, \ldots, N, \quad i \neq j .
$$

The only velocities $v^{i}\left(r^{1}, \ldots, r^{N}\right)$ in (1.12) depend on $n, k$. They are described by $k+2$ linearly independent solutions of the linear system (1.8), (1.9) (see Section 5, Theorem 3). One has 
to substitute functions $u_{1}=u_{1}\left(r^{1}, \ldots, r^{N}\right), \ldots, u_{n}=u_{n}\left(r^{1}, \ldots, r^{N}\right)$ for the arguments of these solutions. The functions $u_{i}$ are also universal. They are defined by the following system of PDEs

$$
\partial_{i} u_{j}=\frac{u_{j}\left(u_{j}-1\right) \partial_{i} w}{\xi_{i}-u_{j}}, \quad i=1, \ldots, N, \quad j=1, \ldots, n .
$$

It is easy to verify that the system (1.13), (1.14) is consistent. Therefore our $(1+1)$-dimensional systems (1.12) admit a local parametrization by $2 N$ functions of one variable.

For some very special values of parameters $s_{i}$ in (1.8), (1.9) our systems (1.12) are related to the Whitham hierarchies [2, to the Frobenious manifolds [3, 16], and to the associativity equation [3, 16].

In Section 6 we recall the definition of hydrodynamic reductions. According to [17], the existence of sufficiently many hydrodynamic reductions can be chosen as a definition of the integrability of the systems (1.5). We also recall the definition of integrable pseudopotentials (see [7]). We introduce the notion of compatible pseudopotentials and notice that each pair of them gives a system (1.5) that admits both a pseudopotential representation and sufficiently many hydrodynamic reductions. We show that the $(1+1)$-dimensional hydrodynamic type systems found in Section 5 are hydrodynamic reductions of our pseudopotentials $A_{n, k}$. This implies that these pseudopotentials and the corresponding 3-dimensional systems are integrable in the sense of the definitions mentioned above (see Theorem 4).

\section{Generalized hypergeometric functions}

The following statements can be verified straightforwardly.

Proposition 1. The system of linear equations (1.8), (1.9) is compatible for any constants $s_{1}, \ldots, s_{n+2}$. The dimension of the linear space $\mathcal{H}$ of solutions of the system (1.8), (1.9) is equal to $n+1$.

We call elements of $\mathcal{H}$ generalized hypergeometric functions.

Proposition 2. The system (1.8), (1.9) is equivalent to the following system

$$
Q_{i}\left(u_{1} \frac{\partial}{\partial u_{1}}, \ldots, u_{n} \frac{\partial}{\partial u_{n}}\right) u_{i}^{-1} h=P_{i}\left(u_{1} \frac{\partial}{\partial u_{1}}, \ldots, u_{n} \frac{\partial}{\partial u_{n}}\right) h, \quad i=1, \ldots, n
$$

where

$$
\begin{gathered}
Q_{i}\left(k_{1}, \ldots, k_{n}\right)=\left(k_{1}+\ldots+k_{n}-s_{1}-\ldots-s_{n+1}\right)\left(k_{i}+1\right), \\
P_{i}\left(k_{1}, \ldots, k_{n}\right)=\left(k_{1}+\ldots+k_{n}-1-s_{1}-\ldots-s_{n+2}\right)\left(k_{i}-s_{i}\right) .
\end{gathered}
$$

Recall that a system of the form (2.15) is called a hypergeometric system [9]. It can be solved in terms of the so-called Horn series [9]. 
Example 1. The system (2.15) and hence (1.8), (1.9) has a unique solution holomorphic at the point $\mathbf{0}=(0, \ldots, 0)$ such that $h(\mathbf{0})=1$. The derivatives of this solution at $\mathbf{0}$ is given by

$$
h^{\left(k_{1}, \ldots, k_{n}\right)}(\mathbf{0})=\frac{\prod_{j=0}^{k_{1}+\ldots+k_{n}-1}\left(1-j+s_{n+2}+r\right)}{\prod_{j=0}^{k_{1}+\ldots+k_{n}-1}(-j+r)} \prod_{j=1}^{n} \prod_{i=0}^{k_{j}-1}\left(i-s_{j}\right)
$$

where

$$
r=\sum_{i=1}^{n+1} s_{i}
$$

Let us denote the solution described in this example by $F\left(s_{1}, \ldots, s_{n+2}, u_{1}, \ldots, u_{n}\right)$. For brevity, we also will use the notation $F\left(s_{1}, \ldots, s_{n+2}\right)$.

Proposition 3. The function $F\left(s_{1}, \ldots, s_{n+2}\right)$ admits the following integral representation

$$
F\left(s_{1}, \ldots, s_{n+2}, u_{1}, \ldots, u_{n}\right)=C \int_{0}^{1} t^{-2-r-s_{n+2}}(1-t)^{s_{n+2}}\left(1-t u_{1}\right)^{s_{1}} \cdots\left(1-t u_{n}\right)^{s_{n}} d t
$$

where

$$
C=\frac{\Gamma(-r)}{\Gamma\left(1+s_{n+2}\right) \Gamma\left(-1-r-s_{n+2}\right)} .
$$

It is well-known that for the standard hypergeometric equation there exist the Laplace transformations shifting the parameters by 1. Analogies of such transformations for the system (1.8), (1.9) are given by

Proposition 4. The following identities hold:

$$
\begin{gathered}
\frac{\partial F\left(s_{1}, \ldots, s_{i}, \ldots s_{n+2}\right)}{\partial u_{i}}=-\frac{s_{i}\left(1+r+s_{n+2}\right)}{r} F\left(s_{1}, \ldots, s_{i}-1, \ldots s_{n+2}\right), \quad i \leq n \\
L_{1}\left(F\left(s_{1}, \ldots, s_{n+1}, s_{n+2}\right)\right)=\frac{s_{n+1}\left(1+r+s_{n+2}\right)}{r} F\left(s_{1}, \ldots, s_{n+1}-1, s_{n+2}\right) \\
L_{2}\left(F\left(s_{1}, \ldots, s_{n+1}, s_{n+2}\right)\right)=\left(1+r+s_{n+2}\right) F\left(s_{1}, \ldots, s_{n+1}, s_{n+2}-1\right)
\end{gathered}
$$

where

$$
L_{1}=\sum_{j=1}^{n}\left(1-u_{j}\right) \frac{\partial}{\partial u_{j}}+\left(1+r+s_{n+2}\right), \quad L_{2}=-\sum_{j=1}^{n} u_{j} \frac{\partial}{\partial u_{j}}+\left(1+r+s_{n+2}\right),
$$

and

$$
\begin{gathered}
M_{i}\left(F\left(s_{1}, \ldots, s_{i}, \ldots s_{n+2}\right)\right)=(1+r) F\left(s_{1}, \ldots, s_{i}+1, \ldots s_{n+2}\right), \quad i \leq n \\
M_{n+1}\left(F\left(s_{1}, \ldots, s_{n+1}, s_{n+2}\right)\right)=(1+r) F\left(s_{1}, \ldots, s_{n+1}+1, s_{n+2}\right)
\end{gathered}
$$




$$
M_{n+2}\left(F\left(s_{1}, \ldots, s_{n+1}, s_{n+2}\right)\right)=-\left(1+s_{n+2}\right) F\left(s_{1}, \ldots, s_{n+1}, s_{n+2}+1\right),
$$

where

$$
\begin{gathered}
M_{i}=\sum_{j=1}^{n} u_{j}\left(u_{j}-1\right) \frac{\partial}{\partial u_{j}}-\sum_{j=1}^{n} s_{j} u_{j}-\left(2+r+s_{n+2}\right) u_{i}+(1+r), \quad i \leq n, \\
M_{n+1}=\sum_{j=1}^{n} u_{j}\left(u_{j}-1\right) \frac{\partial}{\partial u_{j}}-\sum_{j=1}^{n} s_{j} u_{j}+(1+r), \\
M_{n+2}=\sum_{j=1}^{n} u_{j}\left(u_{j}-1\right) \frac{\partial}{\partial u_{j}}-\sum_{j=1}^{n} s_{j} u_{j}-\left(1+s_{n+2}\right) .
\end{gathered}
$$

Furthermore, let $\mathcal{H}_{s_{1}, \ldots, s_{n+2}}$ be the space of solutions of the system (1.8), (1.9). We have

$$
\begin{aligned}
\frac{\partial}{\partial u_{i}} \mathcal{H}_{s_{1}, \ldots, s_{n+2}} & \subset \mathcal{H}_{s_{1}, \ldots, s_{i}-1, \ldots, s_{n+2}}, & L_{1} \mathcal{H}_{s_{1}, \ldots, s_{n+2}} \subset \mathcal{H}_{s_{1}, \ldots, s_{n+1}-1, s_{n+2}}, \\
L_{2} \mathcal{H}_{s_{1}, \ldots, s_{n+2}} & \subset \mathcal{H}_{s_{1}, \ldots, S_{n+2}-1}, & M_{i} \mathcal{H}_{s_{1}, \ldots, s_{n+2}} \subset \mathcal{H}_{s_{1}, \ldots, s_{i}+1, \ldots, s_{n+2}} .
\end{aligned}
$$

Proposition 5. Let $\mathcal{H}=\mathcal{H}_{s_{1}, \ldots, s_{n+2}}$ and $\widetilde{\mathcal{H}}=\mathcal{H}_{s_{1}, \ldots, s_{n}, 0, s_{n+1}, s_{n+2}}$. Then $\widetilde{\mathcal{H}}$ is spanned by $\mathcal{H}$ and the function

$$
Z\left(u_{1}, \ldots, u_{n}, u_{n+1}\right)=\int_{0}^{u_{n+1}}\left(t-u_{1}\right)^{s_{1}} \cdots\left(t-u_{n}\right)^{s_{n}} t^{s_{n+1}}(t-1)^{s_{n+2}} d t .
$$

Moreover, the space $\mathcal{H}_{s_{1}, \ldots, s_{n}, 0, \ldots, 0, s_{n+1}, s_{n+2}}(m$ zeros $)$ is spanned by $\mathcal{H}$ and $Z\left(u_{1}, \ldots, u_{n}, u_{n+1}\right)$, $Z\left(u_{1}, \ldots, u_{n}, u_{n+2}\right), \ldots, Z\left(u_{1}, \ldots, u_{n}, u_{n+m}\right)$.

\section{Pseudopotentials of defect 0}

Most results of this Section was obtained in a different form in the paper [5].

For any generalized hypergeometric function $g \in \mathcal{H}$ we put

$$
S_{n}(g, \xi)=\sum_{1 \leq i \leq n} u_{i}\left(u_{i}-1\right)\left(\xi-u_{1}\right) \ldots \hat{i} \ldots\left(\xi-u_{n}\right) g_{u_{i}}+\left(1+\sum_{1 \leq i \leq n+2} s_{i}\right)\left(\xi-u_{1}\right) \ldots\left(\xi-u_{n}\right) g
$$

Here $g_{u_{i}}=\frac{\partial g}{\partial u_{i}}$. It is clear that $S_{n}(g, \xi)$ is a polynomial of degree $n$ in $\xi$.

Example 2. In the simplest case $n=1$ we have

$$
S_{1}(g, \xi)=u(u-1) g_{u}+\left(1+s_{1}+s_{2}+s_{3}\right)(\xi-u) g
$$


where $u=u_{1}$.

We need the following property of the polynomial $S_{n}(g, \xi)$ :

Lemma 1. For any $1 \leq m \leq n$ the following identity is valid

$$
\begin{gathered}
u_{m}\left(u_{m}-1\right)\left(u_{m}-u_{1}\right) \ldots \hat{m} \ldots\left(u_{m}-u_{n}\right) \frac{S_{n}(g, \xi)_{u_{m}}+\frac{\left(s_{m}+1\right) S_{n}(g, \xi)}{\xi-u_{m}}}{S_{n}\left(g, u_{m}\right)}= \\
\xi(\xi-1)\left(\xi-u_{1}\right) \ldots \hat{m} \ldots\left(\xi-u_{n}\right)\left(\frac{s_{1}}{\xi-u_{1}}+\ldots+\frac{s_{m}+1}{\xi-u_{m}}+\ldots+\frac{s_{n}}{\xi-u_{n}}+\frac{s_{n+1}}{\xi}+\frac{s_{n+2}}{\xi-1}\right) .
\end{gathered}
$$

Define $P_{n}(g, \xi)$ by the formula

$$
P_{n}(g, \xi)=\int_{0}^{\xi} S_{n}(g, \xi)\left(\xi-u_{1}\right)^{-s_{1}-1} \ldots\left(\xi-u_{n}\right)^{-s_{n}-1} \xi^{-s_{n+1}-1}(\xi-1)^{-s_{n+2}-1} d \xi
$$

if $\operatorname{Re} s_{n+1}<-1$ and as the analytic continuation of this expression otherwise.

Proposition 6. The expression

$$
\frac{P_{n}(g, \xi)_{u_{m}}}{S_{n}\left(g, u_{m}\right)}
$$

does not depend on $g$. More precisely,

$$
\begin{gathered}
u_{m}\left(u_{m}-1\right)\left(u_{m}-u_{1}\right) \ldots \hat{m} \ldots\left(u_{m}-u_{n}\right) \frac{P_{n}(g, \xi)_{u_{m}}}{S_{n}\left(g, u_{m}\right)}= \\
-\left(\xi-u_{1}\right)^{-s_{1}} \ldots\left(\xi-u_{m}\right)^{-s_{m}-1} \ldots\left(\xi-u_{n}\right)^{-s_{n}} \xi^{-s_{n+1}}(\xi-1)^{-s_{n+2}} .
\end{gathered}
$$

Proof. The derivative of $(3.19)$ with respect to $\xi$ is equal to

$$
\frac{S_{n}(g, \xi)_{u_{m}}+\frac{\left(s_{m}+1\right) S_{n}(g, \xi)}{\xi-u_{m}}}{S_{n}\left(g, u_{m}\right)}\left(\xi-u_{1}\right)^{-s_{1}-1} \ldots\left(\xi-u_{n}\right)^{-s_{n}-1} \xi^{-s_{n+1}-1}(\xi-1)^{-s_{n+2}-1} .
$$

Lemma 1 implies that this derivative does not depend on $g$. Since the value of (3.19) at $\xi=0$ is equal to zero, expression (3.19) itself does not depend of $g$. Identity (3.20) also follows from Lemma 1.

Let $g_{1}, g_{0}$ be linearly independent elements of $\mathcal{H}$. A pseudopotential $A_{n}\left(p, u_{1}, \ldots, u_{n}\right)$ defined in a parametric form by

$$
A_{n}=P_{n}\left(g_{1}, \xi\right), \quad p=P_{n}\left(g_{0}, \xi\right)
$$

is called pseudopotential of defect 0 .

Relations (3.21) mean that to find $A_{n}\left(p, u_{1}, \ldots, u_{n}\right)$, we have to express $\xi$ from the second equation and substitute the result into the first equation. 
Let $g_{0}, g_{1}, \ldots, g_{n} \in \mathcal{H}$ be a basis in $\mathcal{H}$. Define pseudopotentials $B_{\alpha}\left(p, u_{1}, \ldots, u_{n}\right)$ of defect 0 , where $\alpha=1, \ldots, n$, by

$$
B_{\alpha}=P_{n}\left(g_{\alpha}, \xi\right), \quad p=P_{n}\left(g_{0}, \xi\right), \quad \alpha=1, \ldots, n
$$

Suppose that $u_{1}, \ldots, u_{n}$ are functions of $t_{0}=x, t_{1}, \ldots, t_{n}$.

Theorem 1. The compatibility conditions $\psi_{t_{\alpha} t_{\beta}}=\psi_{t_{\beta} t_{\alpha}}$ for the system

$$
\psi_{t_{\alpha}}=B_{\alpha}\left(\psi_{x}, u_{1}, \ldots, u_{n}\right), \quad \alpha=1, \ldots, n .
$$

are equivalent to the following system of PDEs for $u_{1}, \ldots, u_{n}$ :

$$
\begin{gathered}
\sum_{1 \leq i \leq n, i \neq j}\left(g_{q, u_{j}} g_{r, u_{i}}-g_{r, u_{j}} g_{q, u_{i}}\right) \frac{u_{j}\left(u_{j}-1\right) u_{i, t_{s}}-u_{i}\left(u_{i}-1\right) u_{j, t_{s}}}{u_{j}-u_{i}}+\sigma \cdot\left(g_{q} g_{r, u_{j}}-g_{r} g_{q, u_{j}}\right) u_{j, t_{s}}+ \\
\sum_{1 \leq i \leq n, i \neq j}\left(g_{r, u_{j}} g_{s, u_{i}}-g_{s, u_{j}} g_{r, u_{i}}\right) \frac{u_{j}\left(u_{j}-1\right) u_{i, t_{q}}-u_{i}\left(u_{i}-1\right) u_{j, t_{q}}}{u_{j}-u_{i}}+\sigma \cdot\left(g_{r} g_{s, u_{j}}-g_{s} g_{r, u_{j}}\right) u_{j, t_{q}}+(3.23) \\
\sum_{1 \leq i \leq n, i \neq j}\left(g_{s, u_{j}} g_{q, u_{i}}-g_{q, u_{j}} g_{s, u_{i}}\right) \frac{u_{j}\left(u_{j}-1\right) u_{i, t_{r}}-u_{i}\left(u_{i}-1\right) u_{j, t_{r}}}{u_{j}-u_{i}}+\sigma \cdot\left(g_{s} g_{q, u_{j}}-g_{q} g_{s, u_{j}}\right) u_{j, t_{r}}=0,
\end{gathered}
$$

where $j=1, \ldots, n, \sigma=1+s_{1}+\ldots+s_{n+2}$. Here $q, r, s$ run from 0 to $n$ and $t_{0}=x$.

Proof. If $B_{\alpha}$ are given in a parametric form

$$
B_{\alpha}=f_{\alpha}\left(\xi, u_{1}, \ldots, u_{n}\right), \quad p=f_{0}\left(\xi, u_{1}, \ldots, u_{n}\right),
$$

then the compatibility conditions for (3.22) is equivalent to

$$
\sum_{i=1}^{n}\left(\left(f_{q, \xi} f_{r, u_{i}}-f_{r, \xi} f_{q, u_{i}}\right) u_{i, t_{s}}+\left(f_{r, \xi} f_{s, u_{i}}-f_{s, \xi} f_{r, u_{i}}\right) u_{i, t_{q}}+\left(f_{s, \xi} f_{q, u_{i}}-f_{q, \xi} f_{s, u_{i}}\right) u_{i, t_{r}}\right)=0 .
$$

Taking into account (3.18), (3.20), we get

$$
\begin{gathered}
f_{q, \xi} f_{r, u_{i}}-f_{r, \xi} f_{q, u_{i}}= \\
\left(S_{n}\left(g_{q}, \xi\right) P_{n}\left(g_{r}, \xi\right)_{u_{i}}-S_{n}\left(g_{r}, \xi\right) P_{n}\left(g_{q}, \xi\right)_{u_{i}}\right)\left(\xi-u_{1}\right)^{-s_{1}-1} \cdots\left(\xi-u_{n}\right)^{-s_{n}-1} \xi^{-s_{n+1}-1}(\xi-1)^{-s_{n+2}-1} \\
=\frac{S_{n}\left(g_{q}, \xi\right) S_{n}\left(g_{r}, u_{i}\right)-S_{n}\left(g_{r}, \xi\right) S_{n}\left(g_{q}, u_{i}\right)}{\left(\xi-u_{i}\right) \cdot u_{i}\left(u_{i}-1\right)\left(u_{i}-u_{1}\right) \ldots \hat{i} \ldots\left(u_{i}-u_{n}\right)} \cdot T=\frac{S_{n}\left(g_{q}, \xi\right) g_{r, u_{i}}-S_{n}\left(g_{r}, \xi\right) g_{q, u_{i}}}{\xi-u_{i}} \cdot T .
\end{gathered}
$$

Here

$$
T=-\left(\xi-u_{1}\right)^{-2 s_{1}-1} \ldots\left(\xi-u_{n}\right)^{-2 s_{n}-1} \xi^{-2 s_{n+1}-1}(\xi-1)^{-2 s_{n+2}-1}
$$


does not depend on $i$. Using the above formula for $f_{q, \xi} f_{r, u_{i}}-f_{r, \xi} f_{q, u_{i}}$ and similar formulas for $f_{r, \xi} f_{s, u_{i}}-f_{s, \xi} f_{r, u_{i}}, f_{s, \xi} f_{q, u_{i}}-f_{q, \xi} f_{s, u_{i}}$, we can rewrite (3.24) as follows:

$$
\begin{gathered}
\sum_{1 \leq i \leq n}\left(\frac{S_{n}\left(g_{q}, \xi\right) g_{r, u_{i}}-S_{n}\left(g_{r}, \xi\right) g_{q, u_{i}}}{\xi-u_{i}} u_{i, t_{s}}+\frac{S_{n}\left(g_{r}, \xi\right) g_{s, u_{i}}-S_{n}\left(g_{s}, \xi\right) g_{r, u_{i}}}{\xi-u_{i}} u_{i, t_{q}}+\right. \\
\left.\frac{S_{n}\left(g_{s}, \xi\right) g_{q, u_{i}}-S_{n}\left(g_{q}, \xi\right) g_{s, u_{i}}}{\xi-u_{i}} u_{i, t_{r}}\right)=0 .
\end{gathered}
$$

It follows from (3.17) that the left hand side is a polynomial of degree $n-1$ in $\xi$. To conclude the proof, it remains to evaluate this polynomial at $\xi=u_{1}, \ldots, u_{n}$.

Remark 1. Given $t_{1}, t_{2}, t_{3}$, Theorem 1 yields a 3 -dimensional system of the form (1.5) with $l=n$ equations possessing pseudopotential representation.

Remark 2. A system of PDEs for $u_{1}, \ldots, u_{n}$, which is equivalent to compatibility conditions for equations of the form (3.24), was called in [2] a Whitham hierarchy. In the paper [2] I.M. Krichever constructed some Whitham hierarchies related to algebraic curves of arbitrary genus $g$. The hierarchy corresponding to $g=0$ is equivalent to one described by Theorem 1 if $s_{1}=\ldots=s_{n+2}=0$. In this case the vector space $\mathcal{H}$ is spanned by $1, u_{1}, u_{2}, \ldots, u_{n}$.

Proposition 7. The system (3.23) possesses $n+1$ hydrodynamic type conservation laws.

Proof. Let $\widehat{\mathcal{H}}=\mathcal{H}_{-2 s_{1}, \ldots,-2 s_{n},-2 s_{n+1}-1,-2 s_{n+2}-1}$ be the space of generalized hypergeometric functions defined by (1.8), (1.9) with $\hat{s}_{i}=-2 s_{i}$ for $i=1, \ldots, n$ and $\hat{s}_{i}=-2 s_{i}-1$ for $i=$

$n+1, n+2$. Let $Z \in \widehat{\mathcal{H}}$ be an arbitrary element in $\widehat{\mathcal{H}}$. Denote by $X_{j}$ the left hand side of (3.23). Define functions $A_{i}, B_{i}, C_{i}$ by

$$
\sum_{i=1}^{n}\left(A_{i} u_{i, t_{q}}+B_{i} u_{i, t_{r}}+C_{i} u_{i, t_{s}}\right)=\sum_{j=1}^{n} \frac{1}{s_{j}} Z_{u_{j}} X_{j} .
$$

One can check that $\left(A_{i}\right)_{u_{j}}=\left(A_{j}\right)_{u_{i}},\left(B_{i}\right)_{u_{j}}=\left(B_{j}\right)_{u_{i}},\left(C_{i}\right)_{u_{j}}=\left(C_{j}\right)_{u_{i}}$. Therefore $A_{i}=A_{u_{i}}$, $B_{i}=B_{u_{i}}, C_{i}=C_{u_{i}}$ for some functions $A, B, C$ and we have

$$
A_{t_{q}}+B_{t_{r}}+C_{t_{s}}=0
$$

Since $\operatorname{dim} \widehat{\mathcal{H}}=n+1$, we obtain $n+1$ conservation laws of the hydrodynamic type.

\section{Pseudopotentials of defect $k>0$}

In this section we construct a new class of pseudopotentials. We call them pseudopotentials of defect $k$. To define pseudopotentials of defect $k$, we fix $k$ linearly independent generalized hypergeometric functions $h_{1}, \ldots, h_{k} \in \mathcal{H}$. For any $g \in \mathcal{H}$ define $S_{n, k}(g, \xi)$ by the formula

$$
S_{n, k}(g, \xi)=\frac{1}{\Delta} \sum_{1 \leq i \leq n-k+1} u_{i}\left(u_{i}-1\right)\left(\xi-u_{1}\right) \ldots \hat{i} \ldots\left(\xi-u_{n-k+1}\right) \Delta_{i}(g) .
$$


Here

$$
\Delta=\operatorname{det}\left(\begin{array}{ccc}
h_{1} & \ldots & h_{k} \\
h_{1, u_{n-k+2}} & \ldots & h_{k, u_{n-k+2}} \\
\ldots \ldots \ldots & \ldots & \ldots \ldots \ldots \\
h_{1, u_{n}} & \ldots & h_{k, u_{n}}
\end{array}\right), \quad \Delta_{i}(g)=\operatorname{det}\left(\begin{array}{cccc}
g & h_{1} & \ldots & h_{k} \\
g_{u_{i}} & h_{1, u_{i}} & \ldots & h_{k, u_{i}} \\
g_{u_{n-k+2}} & h_{1, u_{n-k+2}} & \ldots & h_{k, u_{n-k+2}} \\
\ldots \ldots \ldots & \ldots & \ldots & \ldots \ldots \ldots \\
g_{u_{n}} & h_{1, u_{n}} & \ldots & h_{k, u_{n}}
\end{array}\right) .
$$

It is clear that $S_{n, k}(g, \xi)$ is a polynomial in $\xi$ of degree $n-k$. Notice that $S_{n, k}\left(h_{1}, \xi\right)=\ldots=$ $S_{n, k}\left(h_{k}, \xi\right)=0$. It is easy to see that linear transformations $h_{i} \rightarrow c_{i 1} h_{1}+\ldots+c_{i k} h_{k}, g \rightarrow$ $g+d_{1} h_{1}+\ldots+d_{k} h_{k}$ with constant coefficients $c_{i j}, d_{i}$ do not change $S_{n, k}(g, \xi)$.

Example 3. In the simplest case $n=2, k=1$ we have

$$
S_{2,1}(g, \xi)=u_{1}\left(u_{1}-1\right)\left(\xi-u_{2}\right) \frac{g h_{1, u_{1}}-g_{u_{1}} h_{1}}{h_{1}}+u_{2}\left(u_{2}-1\right)\left(\xi-u_{1}\right) \frac{g h_{1, u_{2}}-g_{u_{2}} h_{1}}{h_{1}} .
$$

Lemma 2. If $1 \leq m<n-k+2$, then the following identity is valid:

$$
\begin{gathered}
u_{m}\left(u_{m}-1\right)\left(u_{m}-u_{1}\right) \ldots \hat{m} \ldots\left(u_{m}-u_{n}\right) \frac{S_{n, k}(g, \xi)_{u_{m}}+\frac{\left(s_{m}+1\right) S_{n, k}(g, \xi)}{\xi-u_{m}}}{S_{n, k}\left(g, u_{m}\right)}= \\
-\left(u_{m}-u_{n-k+2}\right) \ldots\left(u_{m}-u_{n}\right) \frac{1}{\Delta} \sum_{1 \leq i \leq n-k+1} u_{i}\left(u_{i}-1\right)\left(\xi-u_{1}\right) \ldots \hat{i} \ldots\left(\xi-u_{n-k+1}\right) \widetilde{\Delta_{i}}+ \\
\frac{1}{\Delta} \sum_{n-k+2 \leq i \leq n, 1 \leq j \leq n-k+1}\left(u_{m}-u_{n-k+2}\right) \ldots \hat{i} \ldots\left(u_{m}-u_{n}\right) s_{i} u_{j}\left(u_{j}-1\right)\left(\xi-u_{1}\right) \ldots \hat{j} \ldots\left(\xi-u_{n-k+1}\right) \widetilde{\Delta_{i, j}}+ \\
\quad \frac{\left(s_{m}+1\right) u_{m}\left(u_{m}-1\right)\left(u_{m}-u_{n-k+2}\right) \ldots\left(u_{m}-u_{n}\right)\left(\xi-u_{1}\right) \ldots \hat{m} \ldots\left(\xi-u_{n-k+1}\right)}{\xi-u_{m}}+ \\
\quad \sum_{1 \leq i \leq n-k+1, i \neq m} s_{i} u_{i}\left(u_{i}-1\right) \prod_{1 \leq j \leq n-k+1, j \neq i, m}\left(\xi-u_{j}\right)+ \\
\left(u_{m}-u_{n-k+2} \ldots\left(u_{m}-u_{n}\right) \prod_{n+2} \ldots\left(u_{m}-u_{n}\right)\left(\xi-u_{1}\right) \ldots \hat{m} \ldots\left(\xi-u_{n-k+1}\right)\left(\sum_{1 \leq i \leq n-k+1}\left(u_{m}+u_{i}-1\right) s_{i}+2 u_{m}-1\right)+\right. \\
u_{m}\left(u_{m}-1\right)\left(\xi-u_{1}\right) \ldots \hat{m} \ldots\left(\xi-u_{n-k+1} \sum_{n-k+2 \leq i \leq n}\left(u_{m}-u_{n-k+2}\right) \ldots \hat{i} \ldots\left(u_{m}-u_{n}\right) s_{i}+\right. \\
\left(u_{m}-u_{n-k+2}\right) \ldots\left(u_{m}-u_{n}\right)\left(\xi-u_{1}\right) \ldots \hat{m} \ldots\left(\xi-u_{n-k+1}\right)\left(\left(u_{m}-1\right) s_{n+1}+u_{m} s_{n+2}\right) .
\end{gathered}
$$

If $n-k+2 \leq m$, then

$$
\begin{gathered}
u_{m}\left(u_{m}-1\right)\left(u_{m}-u_{1}\right) \ldots \hat{m} \ldots\left(u_{m}-u_{n}\right) \frac{S_{n, k}(g)(\xi)_{u_{m}}+\frac{s_{m} S_{n, k}(g, \xi)}{\xi-u_{m}}}{S_{n, k}\left(g, u_{m}\right)}= \\
\frac{1}{\Delta} \sum_{n-k+2 \leq i \leq n, 1 \leq j \leq n-k+1}\left(u_{m}-u_{n-k+2}\right) \ldots \hat{i} \ldots\left(u_{m}-u_{n}\right) s_{i} u_{j}\left(u_{j}-1\right)\left(\xi-u_{1}\right) \ldots \hat{j} \ldots\left(\xi-u_{n-k+1}\right) \widetilde{\Delta_{i, j}}+
\end{gathered}
$$




$$
\frac{s_{m} u_{m}\left(u_{m}-1\right)\left(u_{m}-u_{n-k+2}\right) \ldots \hat{m} \ldots\left(u_{m}-u_{n}\right)\left(\xi-u_{1}\right) \ldots\left(\xi-u_{n-k+1}\right)}{\xi-u_{m}} .
$$

Here

$$
\widetilde{\Delta_{i}}=\operatorname{det}\left(\begin{array}{ccc}
h_{1, u_{i}} & \ldots & h_{k, u_{i}} \\
h_{1, u_{n-k+2}} & \ldots & h_{k, u_{n-k+2}} \\
\ldots \ldots \ldots & \ldots & \ldots \ldots \ldots \\
h_{1, u_{n}} & \ldots & h_{k, u_{n}}
\end{array}\right)
$$

and $\widetilde{\Delta_{i, j}}$ is obtained from $\Delta$ by replacing the row $\left(h_{1, u_{i}}, \ldots, h_{k, u_{i}}\right)$ by $\left(h_{1, u_{j}}, \ldots, h_{k, u_{j}}\right)$.

Define functions $P_{n, k}(g, \xi)$ by

$$
\begin{gathered}
P_{n, k}(g, \xi)= \\
\int_{0}^{\xi} S_{n, k}(g, \xi)\left(\xi-u_{1}\right)^{-s_{1}-1} \ldots\left(\xi-u_{n-k+1}\right)^{-s_{n-k+1}-1}\left(\xi-u_{n-k+2}\right)^{-s_{n-k+2}} \ldots\left(\xi-u_{n}\right)^{-s_{n}} \xi^{-s_{n+1}-1}(\xi-1)^{-s_{n+2}-1} d \xi
\end{gathered}
$$

if $\operatorname{Re} s_{n+1}<-1$, and as the analytic continuation of this expression otherwise.

Proposition 8. The expression

$$
\frac{P_{n, k}(g, \xi)_{u_{m}}}{S_{n, k}\left(g, u_{m}\right)}
$$

does not depend on $g$. Moreover, we have

$$
\begin{gathered}
\sum_{1 \leq i \leq k+1} u_{m_{i}}\left(u_{m_{i}}-1\right)\left(u_{m_{i}}-u_{1}\right) \ldots m_{1}, \widehat{, ., m}_{k+1} \ldots\left(u_{m_{i}}-u_{n}\right) \frac{P_{n, k}(g, \xi)_{u_{m_{i}}}}{S_{n, k}\left(g, u_{m_{i}}\right)}= \\
-\frac{\left(\xi-u_{1}\right)^{-s_{1}} \ldots\left(\xi-u_{n-k+1}\right)^{-s_{n-k+1}}\left(\xi-u_{n-k+2}\right)^{-s_{n-k+2}+1} \ldots\left(\xi-u_{n}\right)^{-s_{n}+1} \xi^{-s_{n+1}}(\xi-1)^{-s_{n+2}}}{\left(\xi-u_{m_{1}}\right) \ldots\left(\xi-u_{m_{k+1}}\right)} .
\end{gathered}
$$

Proof. The derivative of expression (4.27) with respect to $\xi$ is equal to

$$
\begin{gathered}
\frac{S_{n, k}(g, \xi)_{u_{m}}+\frac{\left(s_{m}+1\right) S_{n, k}(g, \xi)}{\xi-u_{m}}}{S_{n, k}\left(g, u_{m}\right)}\left(\xi-u_{1}\right)^{-s_{1}-1} \ldots \\
\left(\xi-u_{n-k+1}\right)^{-s_{n-k+1}-1}\left(\xi-u_{n-k+2}\right)^{-s_{n-k+2}} \ldots\left(\xi-u_{n}\right)^{-s_{n}} \xi^{-s_{n+1}-1}(\xi-1)^{-s_{n+2}-1}
\end{gathered}
$$

for $1 \leq m<n-k+2$ and is equal to

$$
\begin{gathered}
\frac{S_{n, k}(g, \xi)_{u_{m}}+\frac{s_{m} S_{n, k}(g, \xi)}{\xi-u_{m}}}{S_{n, k}\left(g, u_{m}\right)}\left(\xi-u_{1}\right)^{-s_{1}-1} \ldots \\
\left(\xi-u_{n-k+1}\right)^{-s_{n-k+1}-1}\left(\xi-u_{n-k+2}\right)^{-s_{n-k+2}} \ldots\left(\xi-u_{n}\right)^{-s_{n}} \xi^{-s_{n+1}-1}(\xi-1)^{-s_{n+2}-1}
\end{gathered}
$$

otherwise. Lemma 2 implies that this derivative does not depend on $g$. Moreover, the value of the expression (4.27) at $\xi=0$ is equal to zero. Therefore the expression (4.27) itself does not depend on $g$. The proof of (4.28) is similar. 
Let $g_{1}, g_{2} \in \mathcal{H}$. Assume that $g_{1}, g_{2}, h_{1}, \ldots, h_{k}$ are linearly independent. Define pseudopotential $A_{n, k}\left(p, u_{1}, \ldots, u_{n}\right)$ in parametric form by

$$
A_{n, k}=P_{n, k}\left(g_{1}, \xi\right), \quad p=P_{n, k}\left(g_{2}, \xi\right) .
$$

To construct $A_{n, k}\left(p, u_{1}, \ldots, u_{n}\right)$, we find $\xi$ from the second equation and substitute into the first one. The pseudopotential $A_{n, k}\left(p, u_{1}, \ldots, u_{n}\right)$ is called pseudopotential of defect $k$.

Theorem 2. Let $g_{0}, g_{1}, \ldots, g_{n-k}, h_{1}, \ldots, h_{k} \in \mathcal{H}$ be a basis in $\mathcal{H}$ and $B_{\alpha}, \alpha=1, \ldots, n-k$ are defined by

$$
B_{\alpha}=P_{n, k}\left(g_{\alpha}, \xi\right), \quad p=P_{n, k}\left(g_{0}, \xi\right), \quad \alpha=1, \ldots, n-k .
$$

Then the compatibility conditions for (3.22) are equivalent to the following system of PDEs for $u_{1}, \ldots, u_{n}$ :

$$
\begin{gathered}
\sum_{1 \leq i \leq n-k, i \neq j}\left(\Delta_{j}\left(g_{q}\right) \Delta_{i}\left(g_{r}\right)-\Delta_{j}\left(g_{r}\right) \Delta_{i}\left(g_{q}\right)\right) \frac{u_{j}\left(u_{j}-1\right) u_{i, t_{s}}-u_{i}\left(u_{i}-1\right) u_{j, t_{s}}}{u_{j}-u_{i}}+ \\
\sum_{1 \leq i \leq n-k, i \neq j}\left(\Delta_{j}\left(g_{r}\right) \Delta_{i}\left(g_{s}\right)-\Delta_{j}\left(g_{s}\right) \Delta_{i}\left(g_{r}\right)\right) \frac{u_{j}\left(u_{j}-1\right) u_{i, t_{q}}-u_{i}\left(u_{i}-1\right) u_{j, t_{q}}}{u_{j}-u_{i}}+ \\
\sum_{1 \leq i \leq n-k, i \neq j}\left(\Delta_{j}\left(g_{s}\right) \Delta_{i}\left(g_{q}\right)-\Delta_{j}\left(g_{q}\right) \Delta_{i}\left(g_{s}\right)\right) \frac{u_{j}\left(u_{j}-1\right) u_{i, t_{r}}-u_{i}\left(u_{i}-1\right) u_{j, t_{r}}}{u_{j}-u_{i}}=0,
\end{gathered}
$$

where $j=1, \ldots, n-k$ and

$$
\begin{aligned}
& \sum_{i=1}^{n-k+1} \Delta_{i}\left(g_{r}\right) u_{i, t_{s}}=\sum_{i=1}^{n-k+1} \Delta_{i}\left(g_{s}\right) u_{i, t_{r}} \\
& \sum_{i=1}^{n-k+1} \Delta_{i}\left(g_{r}\right) \frac{u_{m}\left(u_{m}-1\right) u_{i, t_{s}}-u_{i}\left(u_{i}-1\right) u_{m, t_{s}}}{u_{m}-u_{i}}=\sum_{i=1}^{n-k+1} \Delta_{i}\left(g_{s}\right) \frac{u_{m}\left(u_{m}-1\right) u_{i, t_{r}}-u_{i}\left(u_{i}-1\right) u_{m, t_{r}}}{u_{m}-u_{i}},
\end{aligned}
$$

where $m=n-k+2, \ldots, n$. Here $q, r, s$ run from 0 to $n$ and $t_{0}=x$.

Proof. We have to explicitly calculate the coefficients in (3.24). Using (4.26), (4.27), we find that

$$
\begin{gathered}
f_{q, \xi} f_{r, u_{i}}-f_{r, \xi} f_{q, u_{i}}=\left(S_{n, k}\left(g_{q}, \xi\right) P_{n, k}\left(g_{r}, \xi\right)_{u_{i}}-S_{n, k}\left(g_{r}, \xi\right) P_{n, k}\left(g_{q}, \xi\right)_{u_{i}}\right) \cdot T= \\
\left(S_{n, k}\left(g_{q}, \xi\right) S_{n, k}\left(g_{r}, u_{i}\right)-S_{n, k}\left(g_{r}, \xi\right) S_{n, k}\left(g_{q}, u_{i}\right)\right) \cdot \frac{P_{n, k}\left(g_{q}, \xi\right)_{u_{i}}}{\left.S_{n, k}\left(g_{q}, u_{i}\right)\right)} \cdot T .
\end{gathered}
$$

Similar formulas are valid for $f_{r, \xi} f_{s, u_{i}}-f_{s, \xi} f_{r, u_{i}}, f_{s, \xi} f_{q, u_{i}}-f_{q, \xi} f_{s, u_{i}}$. Here

$$
T=\left(\xi-u_{1}\right)^{-s_{1}-1} \ldots\left(\xi-u_{n-k+1}\right)^{-s_{n-k+1}-1}\left(\xi-u_{n-k+2}\right)^{-s_{n-k+2}} \ldots\left(\xi-u_{n}\right)^{-s_{n}} \xi^{-s_{n+1}-1}(\xi-1)^{-s_{n+2}-1}
$$


does not depend on $i$. Using (4.28), we can express $\frac{P_{n, k}\left(g_{q}, \xi\right)_{u_{i}}}{S_{n, k}\left(g_{q}, u_{i}\right)}, i=1, \ldots, n-k$ in terms of $\frac{P_{n, k}\left(g_{q}, \xi\right)_{u_{m}}}{S_{n, k}\left(g_{q}, u_{m}\right)}, m=n-k+1, \ldots, n$, which are linearly independent as functions of $\xi$. Substituting these into (3.24), we obtain

$$
\begin{aligned}
& \sum_{i=1}^{n-k}\left(\frac{S_{n, k}\left(g_{q}, \xi\right) S_{n, k}\left(g_{r}, u_{i}\right)-S_{n, k}\left(g_{r}, \xi\right) S_{n, k}\left(g_{q}, u_{i}\right)}{\left(\xi-u_{i}\right) \cdot u_{i}\left(u_{i}-1\right)\left(u_{i}-u_{1}\right) \ldots \hat{i} \ldots\left(u_{i}-u_{n-k}\right)} u_{i, t_{s}}+\right. \\
& \quad \frac{S_{n, k}\left(g_{r}, \xi\right) S_{n, k}\left(g_{s}, u_{i}\right)-S_{n, k}\left(g_{s}, \xi\right) S_{n, k}\left(g_{r}, u_{i}\right)}{\left(\xi-u_{i}\right) \cdot u_{i}\left(u_{i}-1\right)\left(u_{i}-u_{1}\right) \ldots \hat{i} \ldots\left(u_{i}-u_{n-k}\right)} u_{i, t_{q}}+ \\
& \left.\frac{S_{n, k}\left(g_{s}, \xi\right) S_{n, k}\left(g_{q}, u_{i}\right)-S_{n, k}\left(g_{q}, \xi\right) S_{n, k}\left(g_{s}, u_{i}\right)}{\left(\xi-u_{i}\right) \cdot u_{i}\left(u_{i}-1\right)\left(u_{i}-u_{1}\right) \ldots \hat{i} \ldots\left(u_{i}-u_{n-k}\right)} u_{i, t_{r}}\right)=0 \\
& \sum_{i=1}^{n-k}\left(\frac{S_{n, k}\left(g_{q}, \xi\right) S_{n, k}\left(g_{r}, u_{i}\right)-S_{n, k}\left(g_{r}, \xi\right) S_{n, k}\left(g_{q}, u_{i}\right)}{\left(u_{i}-u_{m}\right) \cdot u_{i}\left(u_{i}-1\right)\left(u_{i}-u_{1}\right) \ldots \hat{i} \ldots\left(u_{i}-u_{n-k}\right)} u_{i, t_{s}}+\right. \\
& \frac{S_{n, k}\left(g_{r}, \xi\right) S_{n, k}\left(g_{s}, u_{i}\right)-S_{n, k}\left(g_{s}, \xi\right) S_{n, k}\left(g_{r}, u_{i}\right)}{\left(u_{i}-u_{m}\right) \cdot u_{i}\left(u_{i}-1\right)\left(u_{i}-u_{1}\right) \ldots \hat{i} \ldots\left(u_{i}-u_{n-k}\right)} u_{i, t_{q}}+ \\
& \left.\frac{S_{n, k}\left(g_{s}, \xi\right) S_{n, k}\left(g_{q}, u_{i}\right)-S_{n, k}\left(g_{q}, \xi\right) S_{n, k}\left(g_{s}, u_{i}\right)}{\left(u_{i}-u_{m}\right) \cdot u_{i}\left(u_{i}-1\right)\left(u_{i}-u_{1}\right) \ldots \hat{i} \ldots\left(u_{i}-u_{n-k}\right)} u_{i, t_{r}}\right)+ \\
& \quad \frac{S_{n, k}\left(g_{q}, \xi\right) S_{n, k}\left(g_{r}, u_{m}\right)-S_{n, k}\left(g_{r}, \xi\right) S_{n, k}\left(g_{q}, u_{m}\right)}{u_{m}\left(u_{m}-1\right)\left(u_{m}-u_{1}\right) \ldots\left(u_{m}-u_{n-k}\right)} u_{m, t_{s}}+ \\
& \frac{S_{n, k}\left(g_{r}, \xi\right) S_{n, k}\left(g_{s}, u_{m}\right)-S_{n, k}\left(g_{s}, \xi\right) S_{n, k}\left(g_{r}, u_{m}\right)}{u_{m}\left(u_{m}-1\right)\left(u_{m}-u_{1}\right) \ldots\left(u_{m}-u_{n-k}\right)} u_{m, t_{q}}+ \\
& \frac{S_{n, k}\left(g_{s}, \xi\right) S_{n, k}\left(g_{q}, u_{m}\right)-S_{n, k}\left(g_{q}, \xi\right) S_{n, k}\left(g_{s}, u_{m}\right)}{u_{m}\left(u_{m}-1\right)\left(u_{m}-u_{1}\right) \ldots\left(u_{m}-u_{n-k}\right)} u_{m, t_{r}}=0
\end{aligned}
$$

where $m=n-k+1, \ldots, n$. One can check straightforwardly that (4.34) is equivalent to (4.31) for $m=n-k+1$ and to (4.32) for $m=n-k+2, \ldots, n$. Notice that the left hand side of equation (4.33) is a polynomial in $\xi$ of degree $n-k-1$. Evaluating this polynomial at $\xi=u_{j}, j=1, \ldots, n-k$ we obtain (4.30).

Remark 3. Given $t_{1}, t_{2}, t_{3}$, Theorem 2 yields a 3-dimensional system of the form (1.5) with $l=n+k$ equations possessing pseudopotential representation. Indeed, the formulas (4.31), (4.32) give $3 k$ linearly independent equations if $q, r, s=1,2,3$. The formula (4.30) gives $n-k$ equations. On the other hand, one can construct exactly $k$ linear combinations of equations (4.31), (4.32) with $q, r, s=1,2,3$ such that derivatives of $u_{i}, i=n-k+1, \ldots, n$ cancel out. Moreover, these linear combinations belong to the span of equations (4.30). Therefore, there exist $(n-k)+3 k-k=n+k$ linearly independent equations.

Remark 4. In (4.30), (4.31), (4.32) we have to assume $n \geq k+2$. Indeed, for $n=k+1$ we cannot construct more then one pseudopotential and therefore there is no any system of 
the form (1.5) associated with this case. However the corresponding pseudopotential generates interesting integrable (1+1)-dimensional systems of hydrodynamic type (see Section 5). Probably these pseudopotentials for $k=0,1, \ldots$ are also related to some infinite integrable chains of the Benney type [18, 19].

The system (4.30)-(4.32) possesses many conservation laws of the hydrodynamic type. In particular, the following statement can be verified by a straightforward calculation.

Proposition 9. For any $r \neq s=0,1, \ldots, n$ there exist $k$ conservation laws for the system (4.30)-(4.32) of the form:

$$
\left(\frac{\Delta\left(g_{r}, h_{1}, \ldots \hat{i} \ldots h_{k}\right)}{\Delta\left(h_{1}, \ldots, h_{k}\right)}\right)_{t_{s}}=\left(\frac{\Delta\left(g_{s}, h_{1}, \ldots \hat{i} \ldots h_{k}\right)}{\Delta\left(h_{1}, \ldots, h_{k}\right)}\right)_{t_{r}}
$$

where $i=1, \ldots, k$. Here

$$
\Delta\left(f_{1}, \ldots, f_{k}\right)=\operatorname{det}\left(\begin{array}{ccc}
f_{1} & \ldots & f_{k} \\
h_{1, u_{n-k+2}} & \cdots & f_{k, u_{n-k+2}} \\
\ldots \ldots \ldots & \cdots & \cdots \cdots \cdots \\
f_{1, u_{n}} & \cdots & f_{k, u_{n}}
\end{array}\right)
$$

Proposition 9 allows us to define functions $z_{1}, \ldots, z_{k}$ such that

$$
\frac{\Delta\left(g_{r}, h_{1}, \ldots \hat{i} \ldots h_{k}\right)}{\Delta\left(h_{1}, \ldots, h_{k}\right)}=z_{i, t_{r}}
$$

for all $i=1, \ldots, k$ and $r=0,1, \ldots, n$.

Suppose $n \geq 3 k$; then the system of the form (1.5) obtained from (4.30), (4.31), (4.32) with $q, r, s=1,2,3$ consists of $3 k$ equations (4.31), (4.32) (they are equivalent to (4.35)) and $n-2 k$ equations of the form (4.30). Indeed, only $n-2 k$ equations (4.30) are linearly independent on (4.31), (4.32). Expressing $u_{1}, \ldots, u_{3 k}$ in terms of $z_{i, t_{1}}, z_{i, t_{2}}, z_{i, t_{3}}, i=1, \ldots, k$ from (4.36) and substituting into $n-2 k$ equations of the form (4.30), we obtain a 3-dimensional system of $n-2 k$ equations for $n-2 k$ unknowns $z_{1}, \ldots, z_{k}, u_{3 k+1}, \ldots u_{n}$. This is a quasi-linear system of the second order with respect to $z_{i}$ and of the first order with respect to $u_{j}$, whose coefficients depend on $z_{i, t_{1}}, z_{i, t_{2}}, z_{i, t_{3}}, i=1, \ldots, k$ and $u_{3 k+1}, \ldots u_{n}$. It is clear that the general solution of the system can be locally parameterized by $n-k$ functions in two variables.

In the case $2 k \leq n<3 k$ the functions $z_{i, t_{1}}, z_{i, t_{2}}, z_{i, t_{3}}, i=1, \ldots, k$ are functionally dependent. We have $3 k-n$ equations of the form

$$
R_{i}\left(z_{1, t_{1}}, z_{1, t_{2}}, z_{1, t_{3}}, \ldots, z_{k, t_{1}}, z_{k, t_{2}}, z_{k, t_{3}}\right)=0, \quad i=1, \ldots, 3 k-n
$$

and $n-2 k$ second order quasi-linear equations. Totally we have $(3 k-n)+(n-2 k)=k$ equations for $k$ unknowns $z_{1}, \ldots, z_{k}$. It is clear that the general solution of this system can be locally parameterized by $n-k$ functions in two variables. 
Suppose $n<2 k$; then we have $n+k<3 k$, which means that $3 k$ equations of the form (4.31), (4.32) are linearly dependent. Probably in this case the general solution of the system can also be locally parameterized by $n-k$ functions in two variables.

One of the most interesting cases is $n=3 k$, when we have a system of $k$ quasi-linear second order equations for the functions $z_{1}, \ldots, z_{k}$. Consider the simplest case $k=1$.

Example 4. In the case $n=3, k=1$ the formulas (4.30), (4.31) can be rewritten as follows. Let $h_{1}, g_{0}, g_{1}, g_{2}$ be linearly independent elements of $\mathcal{H}$. Denote by $B_{i j}$ the cofactors of the matrix

$$
\left(\begin{array}{cccc}
h_{1} & g_{0} & g_{1} & g_{2} \\
h_{1, u_{1}} & g_{0, u_{1}} & g_{1, u_{1}} & g_{1, u_{1}} \\
h_{1, u_{2}} & g_{0, u_{2}} & g_{1, u_{2}} & g_{1, u_{1}} \\
h_{1, u_{3}} & g_{0, u_{3}} & g_{1, u_{3}} & g_{1, u_{3}}
\end{array}\right)
$$

Define vector fields $V_{i}$ by

$$
\begin{aligned}
& V_{1}=B_{22} \frac{\partial}{\partial t_{0}}+B_{23} \frac{\partial}{\partial t_{1}}+B_{24} \frac{\partial}{\partial t_{2}} \\
& V_{2}=B_{32} \frac{\partial}{\partial t_{0}}+B_{33} \frac{\partial}{\partial t_{1}}+B_{34} \frac{\partial}{\partial t_{2}} \\
& V_{3}=B_{42} \frac{\partial}{\partial t_{0}}+B_{43} \frac{\partial}{\partial t_{1}}+B_{44} \frac{\partial}{\partial t_{2}}
\end{aligned}
$$

Then (4.31) is equivalent to

$$
V_{1}\left(u_{2}\right)=V_{2}\left(u_{1}\right), \quad V_{2}\left(u_{3}\right)=V_{3}\left(u_{2}\right), \quad V_{3}\left(u_{1}\right)=V_{1}\left(u_{3}\right) .
$$

Relation (4.30) leads to one more equation

$$
u_{3}\left(u_{3}-1\right)\left(u_{1}-u_{2}\right) V_{1}\left(u_{2}\right)+u_{1}\left(u_{1}-1\right)\left(u_{2}-u_{3}\right) V_{2}\left(u_{3}\right)+u_{2}\left(u_{2}-1\right)\left(u_{3}-u_{1}\right) V_{3}\left(u_{1}\right)=0 \text {. }
$$

The conservation laws (4.35) have the form

$$
\left(\frac{g_{r}}{h_{1}}\right)_{t_{s}}=\left(\frac{g_{s}}{h_{1}}\right)_{t_{r}} .
$$

Introducing $z$ such that $z_{t_{r}}=\frac{g_{r}}{h_{1}}$, we reduce (4.38) to a quasi-linear equation of the form

$$
\sum_{i, j} P_{i, j}\left(z_{t_{0}}, z_{t_{1}}, z_{t_{2}}\right) z_{t_{i}, t_{j}}=0, \quad i, j=0,1,2
$$

In the paper [11] an inexplicit description of all integrable equations (4.39) was proposed. The equation constructed above corresponds to the generic case in this classification. Indeed, it depends on five essential parameters $s_{1}, \ldots, s_{5}$ which agrees with the results of [11].

For integer values of parameters $s_{i}$ equations (1.8), (1.9) can be solved in elementary functions. This provides simple examples of equations (4.39) having pseudopotentials. In the most 
degenerate case $s_{1}=\cdots=s_{5}=0$ one can choose $h_{1}=1, g_{0}=u_{1}, g_{1}=u_{2}, g_{2}=u_{3}$. The corresponding equation (4.39) is given by

$$
z_{t_{2}}\left(z_{t_{2}}-1\right)\left(z_{t_{0}}-z_{t_{1}}\right) z_{t_{0} t_{1}}+z_{t_{0}}\left(z_{t_{0}}-1\right)\left(z_{t_{1}}-z_{t_{2}}\right) z_{t_{1} t_{2}}+z_{t_{1}}\left(z_{t_{1}}-1\right)\left(z_{t_{2}}-z_{t_{0}}\right) z_{t_{2} t_{0}}=0 .
$$

More general examples of equations

$$
P_{1}\left(z_{t_{0}}, z_{t_{1}}, z_{t_{2}}\right) z_{t_{0} t_{1}}+P_{2}\left(z_{t_{0}}, z_{t_{1}}, z_{t_{2}}\right) z_{t_{1} t_{2}}+P_{3}\left(z_{t_{0}}, z_{t_{1}}, z_{t_{2}}\right) z_{t_{2} t_{0}}=0
$$

correspond to $s_{1}=s_{2}=s_{3}=0$. In this case one can choose $h=1, g_{0}=f\left(u_{1}\right), g_{1}=f\left(u_{2}\right)$, $g_{2}=f\left(u_{3}\right)$, where $f^{\prime}(x)=x^{s_{4}}(x-1)^{s_{5}}$. In the new variables $\bar{u}_{i}=f\left(u_{i}\right)$ the system (4.37), (4.38) is equivalent to a single equation of the form (4.40). One of the results of the paper [11] is a complete classification of equations (4.40) possessing a pseudopotential representation. The above example seems to be the generic case in this classification.

The system (4.30)-(4.32) has conservation laws different from (4.35).

Conjecture. The system (4.30) - (4.32) possesses $n+1$ conservation laws of the general form

$$
A_{t_{q}}+B_{t_{r}}+C_{t_{s}}=0
$$

additional to (4.35). This family of conservation laws can be parameterized by elements from $\widehat{\mathcal{H}}=\mathcal{H}_{-2 s_{1}, \ldots,-2 s_{n},-2 s_{n+1}-1,-2 s_{n+2}-1}$ (cf. Proposition 7 ). This conjecture is supported by some computer computations for small $n$ and $k$.

Remark 5. Let us make in (4.26) a change of variables of the form

$$
\xi \rightarrow \frac{a \xi+b}{c \xi+d}, \quad u_{1} \rightarrow \phi_{1}, \ldots, u_{n} \rightarrow \phi_{n}
$$

where $a, b, c, d, \phi_{1}, \ldots, \phi_{n}$ are arbitrary functions in $u_{1}, \ldots, u_{n}$. After that we get under the integral in (4.26) an expression of the form

$$
\begin{gathered}
S(\xi)\left(\xi-\rho_{1}\right)^{-s_{1}-1} \ldots\left(\xi-\rho_{n-k+1}\right)^{-s_{n-k+1}-1}\left(\xi-\rho_{n-k+2}\right)^{-s_{n-k+2}} \ldots\left(\xi-\rho_{n}\right)^{-s_{n}}\left(\xi-\rho_{n+1}\right)^{-s_{n+1}-1} \\
\times\left(\xi-\rho_{n+2}\right)^{-s_{n+2}-1}\left(\xi-\rho_{n+3}\right)^{s_{1}+\ldots+s_{n+2}+1}
\end{gathered}
$$

where $S(\xi)$ is a polynomial in $\xi$ of degree $n-k$ and $\rho_{1}, \ldots, \rho_{n+3}$ are functions of $u_{1}, \ldots, u_{n}$. Therefore the numbers

$$
\left\{-s_{1}-1, \ldots,-s_{n-k+1}-1,-s_{n-k+2}, \ldots,-s_{n},-s_{n+1}-1,-s_{n+2}-1, s_{1}+\ldots+s_{n+2}+1\right\}
$$

play a symmetric role in the constructed pseudopotentials $A_{n, k}$. Using transformations (4.41), one can choose any three of the functions $\rho_{1}, \ldots, \rho_{n+3}$ to be equal to $0,1, \infty$ and the other $n$ functions to be equal to $u_{1}, \ldots, u_{n}$ (cf. [5], Section 3). It would be interesting to study the degenerate cases when some of the functions $\rho_{i}$ coincide (cf. [7], Section 5).

The most symmetric case is given by

$$
s_{1}=\ldots=s_{n-k+1}=s_{n+1}=s_{n+2}=-\frac{k+1}{n+3}, \quad s_{n-k+2}=\ldots=s_{n}=\frac{n-k+2}{n+3} .
$$


In this case all numbers (4.42) are equal to $-\frac{n-k+2}{n+3}$. Possibly for $n=3, k=1$ these values of parameters correspond to pseudopotentials for integrable Lagrangians of the form $\mathcal{L}\left(u_{x}, u_{y}, u_{z}\right)$ [20, 10] whereas for $n=5, k=3$ they are related to the integrable Hirota type equations [13].

Example 5. Let $n=5, k=3$ and $s_{1}=s_{2}=s_{3}=s_{6}=s_{7}=-\frac{1}{2}, s_{4}=s_{5}=\frac{1}{2}$. It turns out that there exists a basis $g_{1}, g_{2}, g_{3}, h_{1}, h_{2}, h_{3}$ in $\mathcal{H}$ such that

$$
\begin{aligned}
& \Delta\left(g_{1}, h_{1}, h_{2}\right)=\Delta\left(g_{3}, h_{2}, h_{3}\right), \\
& \Delta\left(g_{2}, h_{2}, h_{3}\right)=\Delta\left(g_{1}, h_{3}, h_{1}\right), \\
& \Delta\left(g_{3}, h_{3}, h_{1}\right)=\Delta\left(g_{2}, h_{1}, h_{2}\right) .
\end{aligned}
$$

Indeed, the system (4.43) is a consequence of equations

$$
\begin{gathered}
g_{1} h_{1, u_{4}}-h_{1} g_{1, u_{4}}+g_{2} h_{2, u_{4}}-h_{2} g_{2, u_{4}}+g_{3} h_{3, u_{4}}-h_{3} g_{3, u_{4}}=0, \\
g_{1} h_{1, u_{5}}-h_{1} g_{1, u_{5}}+g_{2} h_{2, u_{5}}-h_{2} g_{2, u_{5}}+g_{3} h_{3, u_{5}}-h_{3} g_{3, u_{5}}=0, \\
g_{1, u_{4}} h_{1, u_{5}}-h_{1, u_{4}} g_{1, u_{5}}+g_{2, u_{4}} h_{2, u_{5}}-h_{2, u_{4}} g_{2, u_{5}}+g_{3, u_{4}} h_{3, u_{5}}-h_{3, u_{4}} g_{3, u_{5}}=0 .
\end{gathered}
$$

Consider the system consisting of equations (4.44) and all its first and second derivatives with respect to $u_{1}, \ldots, u_{5}$. Note that differentiating (4.44), we eliminate second derivatives of $h_{i}$ and $g_{i}$ by (1.8), (1.9). One can check that this system is invariant with respect to the derivations by $u_{1}, \ldots, u_{5}$. At a fixed generic point $u_{1}^{0}, \ldots, u_{5}^{0}$ the system can be regaded as an algebraic variety for the values of $g_{i}, h_{i}$ and their first derivatives. It can be checked that this variety consists of several components and the maximal dimension of the component equals 24 . Since the vector fields $\frac{\partial}{\partial u_{i}}$ are tangent to this variety, any its point considered as the initial data defines the solutions $g_{i}, h_{i}$ of (1.8), (1.9) such that the corresponding point belongs to the variety for any values of $u_{1}, \ldots, u_{5}$. It is possible to check that there exists an algebraic component of dimension 21 of the variety such that the Wronskian of $g_{i}, h_{i}$ at $u_{1}^{0}, \ldots, u_{5}^{0}$ is non-zero.

Proposition 9 and equations (4.43) allow us to define a function $z$ such that

$$
\begin{gathered}
z_{t_{1}, t_{1}}=\frac{\Delta\left(g_{1}, h_{2}, h_{3}\right)}{\Delta\left(h_{1}, h_{2}, h_{3}\right)}, z_{t_{2}, t_{2}}=\frac{\Delta\left(g_{2}, h_{3}, h_{1}\right)}{\Delta\left(h_{1}, h_{2}, h_{3}\right)}, z_{t_{3}, t_{3}}=\frac{\Delta\left(g_{3}, h_{1}, h_{2}\right)}{\Delta\left(h_{1}, h_{2}, h_{3}\right)}, \\
z_{t_{1}, t_{2}}=\frac{\Delta\left(g_{2}, h_{2}, h_{3}\right)}{\Delta\left(h_{1}, h_{2}, h_{3}\right)}=\frac{\Delta\left(g_{1}, h_{3}, h_{1}\right)}{\Delta\left(h_{1}, h_{2}, h_{3}\right)}, z_{t_{2}, t_{3}}=\frac{\Delta\left(g_{3}, h_{3}, h_{1}\right)}{\Delta\left(h_{1}, h_{2}, h_{3}\right)}=\frac{\Delta\left(g_{2}, h_{1}, h_{2}\right)}{\Delta\left(h_{1}, h_{2}, h_{3}\right)}, \\
z_{t_{3}, t_{1}}=\frac{\Delta\left(g_{1}, h_{1}, h_{2}\right)}{\Delta\left(h_{1}, h_{2}, h_{3}\right)}=\frac{\Delta\left(g_{2}, h_{1}, h_{2}\right)}{\Delta\left(h_{1}, h_{2}, h_{3}\right)} .
\end{gathered}
$$

It terms of this function we can rewrite the system (4.30), (4.31), (4.32) as a single equation of the form (1.11). Integrable systems of this form were studied in [13]. The pseudopotentials considered above correspond to the generic integrable system of this form.

Remark 6. It is easy to see that the group $S P_{6}$ acts on the set of bases in $\mathcal{H}$ satisfying (4.44). This agrees with the result of [13] that this group acts on the set of integrable equations of the form (1.11). 


\section{$5 \quad$ Integrable $(1+1)$-dimensional systems of hydrodynamic type}

In this section we consider integrable $(1+1)$-dimensional hydrodynamic type systems (1.12) constructed in terms of generalized hypergeometric functions. These systems appear as the so-called hydrodynamic reductions of pseudopotentials $A_{n, k}$ (see the next Section). By integrability we mean the existence of infinite number of hydrodynamic commuting flows and conservation laws. It is known [21] that this is equivalent to the following relations for the velocities $v^{i}\left(r^{1}, \ldots, r^{N}\right)$ :

$$
\partial_{j} \frac{\partial_{i} v^{k}}{v^{i}-v^{k}}=\partial_{i} \frac{\partial_{j} v^{k}}{v^{j}-v^{k}}, \quad i \neq j \neq k,
$$

Here $\partial_{\alpha}=\frac{\partial}{\partial r^{i}}, \alpha=1, \ldots, N$. The system (1.12) is called semi-Hamiltonian if conditions (5.45) hold.

The main geometrical object related to a semi-Hamiltonian system (1.12) is a diagonal metric $g_{k k}, k=1, \ldots, N$, where

$$
\frac{1}{2} \partial_{i} \log g_{k k}=\frac{\partial_{i} v^{k}}{v^{i}-v^{k}}, \quad i \neq k
$$

In view of (5.45), the overdetermined system (5.46) is compatible and the function $g_{k k}$ is defined up to arbitrary factor $\eta_{k}\left(r^{k}\right)$. The metric $g_{k k}$ is called the metric associated to (1.12). It is known that two hydrodynamic type systems are compatible iff they possess a common associated metric [21].

A diagonal metric $g_{k k}$ is called a metric of Egorov type if for any $i, j$

$$
\partial_{i} g_{j j}=\partial_{j} g_{i i}
$$

Note that if a Egorov-type metric associated with a hydrodynamic-type system of the form (1.12) exists, then it is unique. For any Egorov's metric there exists a potential $G$ such that $g_{i i}=$ $\partial_{i} G$. Semi-Hamiltonian systems possessing associated metrics of Egorov type play important role in the theory of WDVV associativity equations and in the theory of Frobenious manifolds [3, 16, 22].

Let $w\left(r^{1}, \ldots, r^{N}\right), \xi_{1}\left(r^{1}, \ldots, r^{N}\right), \ldots, \xi_{N}\left(r^{1}, \ldots, r^{N}\right)$ be a solution of (1.13). It can be easily verified that this system is in involution and therefore its solution admits a local parameterization by $2 N$ functions of one variable. Let $u_{1}\left(r^{1}, \ldots, r^{N}\right), \ldots, u_{n}\left(r^{1}, \ldots, r^{N}\right)$ be a set of solutions of the system (1.14). It is easy to verify that this system is in involution and therefore has an one-parameter family of solutions for fixed $\xi_{i}, w$.

Consider the following system

$$
r_{t}^{i}=\frac{S_{n, k}\left(g_{1}, \xi_{i}\right)}{S_{n, k}\left(g_{2}, \xi_{i}\right)} r_{x}^{i}
$$


where $g_{1}, g_{2}$ are linearly independent solutions of (1.8), (1.9), the polynomials $S_{n, k}, k>0$ are defined by (4.25), and $S_{n, 0}=S_{n}$ (see formula (3.17)).

Theorem 3. The system (5.48) is semi-Hamiltonian. The associated metric is given by

$$
g_{i i}=S_{n}\left(g_{2}, \xi_{i}\right)^{2}\left(\xi_{i}-u_{1}\right)^{-2 s_{1}-2} \cdots\left(\xi_{i}-u_{n}\right)^{-2 s_{n}-2} \xi_{i}^{-2 s_{n+1}-1}\left(\xi_{i}-1\right)^{-2 s_{n+2}-1} \partial_{i} w
$$

for $k=0$, and by

$$
\begin{gathered}
g_{i i}=S_{n, k}\left(g_{2}, \xi_{i}\right)^{2}\left(\xi_{i}-u_{1}\right)^{-2 s_{1}-2} \cdots\left(\xi_{i}-u_{n-k+1}\right)^{-2 s_{n-k+1}-2} \times \\
\left(\xi_{i}-u_{n-k+2}\right)^{-2 s_{n-k+2}} \cdots\left(\xi_{i}-u_{n}\right)^{-2 s_{n}} \xi_{i}^{-2 s_{n+1}-1}\left(\xi_{i}-1\right)^{-2 s_{n+2}-1} \partial_{i} w
\end{gathered}
$$

for $k>0$.

Proof. Substituting the expression for the metric into (5.46), where $v^{i}$ are specified by (5.48), one obtains the identity by virtue of (1.13), (1.14).

Remark 7. The system (5.48) does not possess the associated metric of the Egorov type in general. However, for very special values of the parameters $s_{i}$ in (1.8), (1.9) there exists $g_{2} \in \mathcal{H}$ such that the metric is of the Egorov type for all solutions of the system (1.13), (1.14). For instance, if the defect $k$ equals zero, then this happens exactly in the following cases:

$s_{i}=0$ for all $i$

$s_{l}=-1$ for some $l$ and $s_{i}=0$ for $i \neq l$;

$s_{l}=-\frac{1}{2}$ for some $l$ and $s_{i}=0$ for $i \neq l$;

$s_{j}=s_{l}=-\frac{1}{2}$ for some $j \neq l$ and $s_{i}=0$ for $i \neq j, i \neq l$.

Proposition 10. Suppose that a solution $\xi_{i}, w$ of (1.13) and solutions $u_{1}, \ldots, u_{n}$ of (1.14) are fixed. Then the hydrodynamic type systems

$$
r_{t_{1}}^{i}=\frac{S_{n, k}\left(g_{1}, \xi_{i}\right)}{S_{n, k}\left(g_{3}, \xi_{i}\right)} r_{x}^{i}, \quad r_{t_{2}}^{i}=\frac{S_{n, k}\left(g_{2}, \xi_{i}\right)}{S_{n, k}\left(g_{3}, \xi_{i}\right)} r_{x}^{i}
$$

are compatible for all $g_{1}, g_{2}$.

Proof. Indeed, the metric associated with (5.48) does not depend on $g_{2}$. Therefore the systems (5.49) has a common metric depending on $g_{3}$ and on solutions of (1.13), (1.14).

Remark 8. One can also construct some compatible systems of the form (5.49) using Proposition 5. Set $g_{2}=Z\left(u_{1}, \ldots, u_{n}, u_{n+1}\right)$ in (5.49). Here $u_{n+1}$ is an arbitrary solution of (1.14) distinct from $u_{1}, \ldots, u_{n}$. It is clear that the flows (5.49) are compatible for such $g_{2}$ and any $g_{1} \in \mathcal{H}$. Moreover, Proposition 5 implies that the flows (5.49) are compatible if we set $g_{1}=Z\left(u_{1}, \ldots, u_{n}, u_{n+1}\right), g_{2}=Z\left(u_{1}, \ldots, u_{n}, u_{n+2}\right)$ for two arbitrary solutions $u_{n+1}, u_{n+2}$ of (1.14).

All members of the hierarchy constructed in Proposition 10 possess a dispersionless Lax representation (1.2) with common $L\left(p, r^{1}, \ldots, r^{N}\right)$. Define a function $L\left(\xi, r^{1}, \ldots, r^{N}\right)$ by the following system

$$
\partial_{i} L=\frac{\xi(\xi-1) \partial_{i} w L_{\xi}}{\xi-\xi_{i}}, \quad i=1, \ldots, N
$$


Note that the system (5.50) is in involution and therefore the function $L$ is defined uniquely up to inessential transformations $L \rightarrow \lambda(L)$. To find the function $L\left(p, r^{1}, \ldots, r^{N}\right)$ one has to express $\xi$ in terms of $p$ by (3.21) for $k=0$ or by (4.29) for $k>0$.

Proposition 11. Let $u_{1}, \ldots, u_{n}$ be arbitrary solution of (1.14). Then system (5.48) admits the dispersionless Lax representation (1.2), where $A=A_{n, k}$ is defined by (3.21) for $k=0$ and by (4.29) for $k>0$.

Proof. Substituting $A=A_{n, k}$ defined by (3.21) for $k=0$ and by (4.29) for $k>0$ into (1.2) and calculating $L_{t}$ by virtue of (5.48) we arrive to the expression

$$
\partial_{i} L=\frac{\partial_{i} P_{n, k}\left(g_{2}, \xi\right) \cdot S_{n, k}\left(g_{1}, \xi_{i}\right)-\partial_{i} P_{n, k}\left(g_{1}, \xi\right) \cdot S_{n, k}\left(g_{2}, \xi_{i}\right)}{P_{n, k}\left(g_{2}, \xi\right)_{\xi} \cdot S_{n, k}\left(g_{1}, \xi_{i}\right)-P_{n, k}\left(g_{1}, \xi\right)_{\xi} \cdot S_{n, k}\left(g_{2}, \xi_{i}\right)} L_{\xi}
$$

Taking into account the equation $P_{n, k}\left(g_{i}, \xi\right)_{\xi}=S_{n, k}\left(g_{i}, \xi\right)\left(\xi-u_{1}\right)^{-s_{1}-1} \ldots\left(\xi-u_{n-k+1}\right)^{-s_{n-k+1}-1}(\xi-$ $\left.u_{n-k+2}\right)^{-s_{n-k+2}} \ldots\left(\xi-u_{n}\right)^{-s_{n}} \xi^{-s_{n+1}-1}(\xi-1)^{-s_{n+2}-1}$ and writing down $P_{n, k}\left(g_{i}, \xi\right)_{u_{m}}$ in terms of $P_{n, k}\left(g_{1}, \xi\right)_{u_{n-k+1}}, \ldots, P_{n, k}\left(g_{1}, \xi\right)_{u_{n}}$ by (4.27), (4.28), one can readily check this equation.

Let the function $\xi\left(L, r^{1}, \ldots, r^{N}\right)$ be inverse to $L\left(\xi, r^{1}, \ldots, r^{N}\right)$. It is easy to check that $u=$ $\xi\left(L, r^{1}, \ldots, r^{N}\right)$, where $L$ plays a role of arbitrary parameter, satisfies (1.14).

As usual, the Lax representation defines conserved densities, common for the whole hierarchy, by formula (1.3). Since our definition of $A_{n, k}$ is parametric, we can reformulate this fact as

Proposition 12. Suppose (5.48) is defined by solutions $u_{1}, \ldots, u_{n}$ of the system (1.14). Let $U$ be any solution of (1.14). Then

$$
\frac{\partial}{\partial t} P_{n, k}\left(g_{2}\left(u_{1}, u_{1}, \ldots, u_{n}\right), U\right)=\frac{\partial}{\partial x} P_{n, k}\left(g_{1}\left(u_{1}, u_{1}, \ldots, u_{n}\right), U\right)
$$

is a conservation law for (5.48).

Since the generic solution $U$ depends on a parameter, we have constructed an one-parametric family of common conservation laws for our hierarchy (5.48) of hydrodynamic type systems.

\section{Hydrodynamic reductions and integrability}

In this section we show that integrable $(1+1)$-dimensional systems constructed in Section 5 define hydrodynamic reductions for pseudopotentials and 3-dimensional systems from Sections 3 and 4.

Following [17, 15, 7], we give a definition of integrability for equations (1.2), (1.4) and (1.5) in terms of hydrodynamic reductions.

Suppose there exists a pair of compatible semi-Hamiltonian hydrodynamic-type systems of the form

$$
r_{t_{1}}^{i}=v_{1}^{i}\left(r^{1}, \ldots, r^{N}\right) r_{x}^{i}, \quad r_{t_{2}}^{i}=v_{2}^{i}\left(r^{1}, \ldots, r^{N}\right) r_{x}^{i}
$$


and functions $u_{i}=u_{i}\left(r^{1}, \ldots, r^{N}\right)$ such that these functions satisfy (1.5) for any solution of (6.51). Then (6.51) is called a hydrodynamic reduction for (1.5).

Definition 1 [17]. A system of the form (1.5) is called integrable if equation (1.2) possesses sufficiently many hydrodynamic reductions for each $N \in \mathbb{N}$. "Sufficiently many" means that the set of hydrodynamic reductions can be locally parameterized by $2 N$ functions of one variable. Note that due to gauge transformations $r^{i} \rightarrow \lambda_{i}\left(r^{i}\right)$ we have $N$ essential functional parameters for hydrodynamic reductions.

Suppose there exists a semi-Hamiltonian hydrodynamic-type system (1.12) and functions $u_{i}=u_{i}\left(r^{1}, \ldots, r^{N}\right), L=L\left(p, r^{1}, \ldots, r^{N}\right)$ such that these functions satisfy dispersionless Lax equation (1.2) for any solution $r^{1}(x, t), \ldots, r^{N}(x, t)$ of the system (1.12). Then (1.12) is called $a$ hydrodynamic reduction for (1.2).

Definition 2 [7]. A dispersionless Lax equation (1.2) is called integrable if equation (1.2) possesses sufficiently many hydrodynamic reductions for each $N \in \mathbb{N}$.

We also call the corresponding pseudopotential $A\left(p, u_{1}, \ldots, u_{n}\right)$ integrable.

Example 6. Let us show that $A=\ln (p-u)$ is integrable. Let $w\left(r^{1}, \ldots, r^{N}\right), p_{i}\left(r^{1}, \ldots, r^{N}\right)$, $i=1, \ldots, N$ be an arbitrary solution of the following system (the so-called Gibbons-Tsarev system [14])

$$
\partial_{j} \xi_{i}=\frac{\partial_{j} w}{\xi_{j}-\xi_{i}}, \quad \partial_{i j} w=\frac{2 \partial_{i} w \partial_{j} w}{\left(\xi_{i}-\xi_{j}\right)^{2}}, \quad i, j=1, \ldots, N, \quad i \neq j .
$$

It is easy to verify that this system is in involution and therefore its general solution admits a local parameterizations by $2 N$ functions of one variable. Define a function $L\left(p, r^{1}, \ldots, r^{N}\right)$ by the following system

$$
\partial_{i} L=\frac{\partial_{i} w L_{p}}{p-\xi_{i}}, \quad i=1, \ldots, N .
$$

This system is in involution and therefore defines the function $L$ uniquely up to inessential transformations $L \rightarrow \lambda(L)$. Finally, let $u\left(r^{1}, \ldots, r^{N}\right)$ be a solution of the system

$$
\partial_{i} u=\frac{\partial_{i} w}{\xi_{i}-u}, \quad i=1, \ldots, N
$$

It is easy to check that the system (6.54) is in involution. It follows from (6.52), (6.53), (6.54) that the system

$$
r_{t}^{i}=\frac{1}{\xi_{i}-u} r_{x}^{i}
$$

is a hydrodynamic reduction of equation (1.2) with $A=\ln (p-u)$.

Remark 9. The standard form for the Gibbons-Tsarev system [15] related to hydrodynamic reductions is given by

$$
\partial_{i} \xi_{j}=F\left(\xi_{i}, \xi_{j}, u_{1}, \ldots, u_{n}\right) \partial_{i} u_{n}, \quad \partial_{i} \partial_{j} u_{n}=H\left(\xi_{i}, \xi_{j}, u_{1}, \ldots, u_{n}\right) \partial_{i} u_{n} \partial_{j} u_{n}, \quad i \neq j
$$




$$
\partial_{i} u_{l}=G_{l}\left(\xi_{i}, u_{1}, \ldots, u_{n}\right) \partial_{i} u_{n}, \quad l<n .
$$

Here $i, j=1, \ldots, N, \quad u_{l}\left(r^{1}, \ldots, r^{N}\right)$ are the functions, which define the reduction, and $\xi_{i}\left(r^{1}, \ldots, r^{N}\right)$ are some auxiliary functions. To bring (6.52), (6.54) to this form, one has to eliminate the additional unknown $w$. The result is given by

$$
\partial_{j} \xi_{i}=\frac{\xi_{i}-u}{\xi_{j}-\xi_{i}} \partial_{j} u, \quad \partial_{i} \partial_{j} u=\frac{\xi_{i}+\xi_{j}-2 u}{\left(\xi_{i}-\xi_{j}\right)^{2}} \partial_{i} u \partial_{j} u .
$$

In this case $n=1, u_{1}=u$. There is the following generalization of (6.56) to the case of arbitrary polynomial $P(x)=a_{3} x^{3}+a_{2} x^{2}+a_{1} x+a_{0}$ and arbitrary $n$ :

$$
\begin{gathered}
\frac{u_{1}-\xi_{i}}{P\left(u_{1}\right)} \partial_{i} u_{1}=\ldots=\frac{u_{n}-\xi_{i}}{P\left(u_{n}\right)} \partial_{i} u_{n}, \quad i=1, \ldots, N, \\
\partial_{i j} u_{n}=\frac{K_{2}\left(\xi_{i}, \xi_{j}\right) u_{n}^{2}+K_{1}\left(\xi_{i}, \xi_{j}\right) u_{n}+K_{0}\left(\xi_{i}, \xi_{j}\right)}{P\left(u_{n}\right)\left(\xi_{i}-\xi_{j}\right)^{2}} \partial_{i} u_{n} \partial_{j} u_{n}, \\
\partial_{i} \xi_{j}=\frac{P\left(\xi_{j}\right)\left(u_{n}-\xi_{i}\right)}{P\left(u_{n}\right)\left(\xi_{i}-\xi_{j}\right)} \partial_{i} u_{n}, \quad i, j=1, \ldots, N, \quad i \neq j,
\end{gathered}
$$

where

$$
\begin{gathered}
K_{2}\left(\xi_{i}, \xi_{j}\right)=2 a_{3}\left(\xi_{i}-\xi_{j}\right)^{2}, \\
K_{1}\left(\xi_{i}, \xi_{j}\right)=-a_{3}\left(\xi_{i}^{2} \xi_{j}+\xi_{i} \xi_{j}^{2}\right)+a_{2}\left(\xi_{i}^{2}+\xi_{j}^{2}-4 \xi_{i} \xi_{j}\right)-a_{1}\left(\xi_{i}+\xi_{j}\right)-2 a_{0}, \\
K_{0}\left(\xi_{i}, \xi_{j}\right)=2 a_{3} \xi_{i}^{2} \xi_{j}^{2}+a_{2}\left(\xi_{i}^{2} \xi_{j}+\xi_{i} \xi_{j}^{2}\right)+a_{1}\left(\xi_{i}^{2}+\xi_{j}^{2}\right)+a_{0}\left(\xi_{i}+\xi_{j}\right) .
\end{gathered}
$$

Using transformations of the form $u_{i} \rightarrow \frac{a u_{i}+b}{c u_{i}+d}, \xi_{i} \rightarrow \frac{a \xi_{i}+b}{c \xi_{i}+d}$, one can put the polynomial $P$ to one of the canonical forms: $P(x)=x(x-1), P(x)=x$, or $P(x)=1$. If $P(x)=1$, then (6.57) with $n=1$ coincides with (6.56). Formulas (1.13), (1.14) are equivalent to (6.57), where $P(x)=x(x-1)$.

Definition 3. Two integrable pseudopotentials $A_{1}, A_{2}$ are called compatible if the system

$$
L_{t_{1}}=\left\{L, A_{1}\right\}, \quad L_{t_{2}}=\left\{L, A_{2}\right\}
$$

possesses sufficiently many hydrodynamic reductions (6.51) for each $N \in \mathbb{N}$.

If $A_{1}, A_{2}$ are compatible, then $A=c_{1} A_{1}+c_{2} A_{2}$ is integrable for any constants $c_{1}, c_{2}$. Indeed, the system

$$
r_{t}^{i}=\left(c_{1} v_{1}^{i}(\mathbf{r})+c_{2} v_{2}^{i}(\mathbf{r})\right) r_{x}^{i}
$$

is a hydrodynamic reduction of (1.2).

Example 7. The functions $A_{1}=\ln \left(p-u_{1}\right)$ and $A_{2}=\ln \left(p-u_{2}\right)$ are compatible. Moreover, $A=c_{1} \ln \left(p-u_{1}\right)+\ldots+c_{n} \ln \left(p-u_{n}\right)$ is integrable for any constants $c_{1}, \ldots, c_{n}$. Indeed, let $w$, $p_{i}$ satisfy (6.52) and $u_{1}, u_{2}$ be two different solutions of (6.54). It is easy to check that the corresponding flows are compatible by virtue of (6.52), (6.53), (6.54). 
Definition 4. By 3-dimensional system associated with compatible functions $A_{1}, A_{2}$ we mean the system of the form (1.5) equivalent to compatibility conditions for the system

$$
\psi_{t_{2}}=A_{1}\left(\psi_{t_{1}}, u_{1}, \ldots, u_{n}\right), \quad \psi_{t_{3}}=A_{2}\left(\psi_{t_{1}}, u_{1}, \ldots, u_{n}\right) .
$$

It is clear that any system associated with a pair of compatible functions possesses sufficiently many hydrodynamic reductions and therefore it is integrable in the sense of Definition 1.

Example 8. Let $A_{1}=\ln (p-u)$ and $A_{2}=\ln (p-v)$. The associated 3-dimensional system has the form

$$
u_{t_{3}}=v_{t_{2}}, \quad v_{t_{1}}-v u_{t_{3}}=u_{t_{1}}-u v_{t_{2}} .
$$

The following statement is a reformulation of Proposition 11.

Theorem 4. The system (5.48) is a hydrodynamic reduction of the pseudopotential $A_{n, k}$ defined by (3.21) if $k=0$ and by (4.29) if $k>0$. Recall that we use the notation $S_{n} \equiv$ $S_{n, 0}, A_{n} \equiv A_{n, 0}, P_{n} \equiv P_{n, 0}$.

Proposition 13. Suppose $g_{1}, g_{2}, g_{3}, h_{1}, \ldots, h_{k} \in \mathcal{H}$ are linearly independent. Define pseudopotentials $A_{1}, A_{2}$ by

$$
A_{1}=P_{n, k}\left(g_{1}, \xi\right), \quad A_{2}=P_{n, k}\left(g_{2}, \xi\right), \quad p=P_{n, k}\left(g_{3}, \xi\right) .
$$

Then $A_{1}$ and $A_{2}$ are compatible.

Proof. Note that the system (1.13), (5.50) does not depend on $g_{1}, g_{2}, g_{3}$ and therefore we have a family of functions $L, \xi_{i}, u_{i}$ which give hydrodynamic reduction of the form (5.48) for both $A_{1}$ and $A_{2}$. Moreover, according to Proposition 10 the flows

$$
r_{t_{1}}^{i}=\frac{S_{n, k}\left(g_{1}, \xi_{i}\right)}{S_{n, k}\left(g_{3}, \xi_{i}\right)} r_{x}^{i}, \quad r_{t_{2}}^{i}=\frac{S_{n, k}\left(g_{2}, \xi_{i}\right)}{S_{n, k}\left(g_{3}, \xi_{i}\right)} r_{x}^{i}
$$

are compatible.

Remark 10. This result implies that 3-dimensional hydrodynamic type systems constructed in Sections 4, 5 possess sufficiently many hydrodynamic reductions.

Remark 11. Using proposition 5, one can construct compatible pseudopotentials depending on different number of $u_{i}$. Indeed, let $g_{1}, g_{3}, h_{1}, \ldots, h_{k} \in \mathcal{H}$ and $g_{2}=Z\left(u_{1}, \ldots, u_{n}, u_{n+1}\right)$. Then $A_{2}$ depends on $u_{1}, \ldots, u_{n}, u_{n+1}$ and $A_{1}$ depends on $u_{1}, \ldots, u_{n}$ only.

\section{Conclusion}

All known integrable pseudopotentials $A\left(p, u_{1}, \ldots, u_{n}\right)$ satisfy the property

$$
P\left(\frac{A_{p p p}}{A_{p p}^{2}}, A_{p}\right)=0,
$$


where $P(x, y)$ is a polynomial in $x, y$ with coefficients depending on $u_{1}, \ldots, u_{n}$. In this sense any pseudopotential $A$ is associated with the algebraic curve $\mathcal{E}=\left\{(x, y) \in \mathbb{C}^{2} ; P(x, y)=0\right\}$. Moreover, compatible pseudopotentials are associated to isomorphic curves. If a 3-dimensional dispersionless system is constructed by two compatible pseudopotentials, then this curve is isomorphic to the so-called spectral curve (see [17]) of the system. In this paper we have constructed a wide class of integrable pseudopotentials associated with rational curves. We believe that all pseudopotentials associated with rational curves can be obtained as a limit from our pseudopotentials. We are going to describe all such limits in a separate paper.

It is known 2] that pseudopotentials associated with curves of higher genus also exist. It is likely that one can describe all pseudopotentials associated with the elliptic curve in a similar manner to the way we have done the rational case in this paper. We are going to consider this problem in the next paper.

Acknowledgments. Authors thank B.A. Dubrovin, E.V. Ferapontov, I.M. Krichever, O.I. Mokhov, and M.V. Pavlov for fruitful discussions. V.S. thanks IHES and A.O. thanks MPIM and IHES for hospitality and financial support. V.S. was partially supported by the RFBR grants 08-01-461 and NS 3472.2008.2.

\section{References}

[1] V.E. Zakharov, A.B. Shabat, Integration of non-linear equations of mathematical physics by the inverse scattering method, Func. Anal. and Appl. 13(3) (1979) 13-22.

[2] I.M. Krichever, The $\tau$-function of the universal Whitham hierarchy, matrix models and topological field theories, Comm. Pure Appl. Math., 47 (1994), no. 4, 437-475.

[3] B.A. Dubrovin, Geometry of 2D topological field theories. In Integrable Systems and Quantum Groups, Lecture Notes in Math. 1620 (1996), 120-348.

[4] A. Odesskii, V. Sokolov, On (2+1)-dimensional hydrodynamic-type systems possessing pseudopotential with movable singularities, to appear in Func. Anal. Appl.

[5] A.V. Odesskii, A family of (2+1)-dimensional hydrodynamic-type systems possessing pseudopotential, arXiv:0704.3577v3 [math. AP], to appear in Selecta Mathematica.

[6] M.V. Pavlov, Classification of the Egorov hydrodynamic chains. Theor. Math. Phys. 138 No. 1 (2004) 55-71.

[7] A. Odesskii, M.V. Pavlov and V.V. Sokolov, Classification of integrable Vlasov-type equations, arXiv:0710.5655, Theor. Math. Phys. 154(2)(2008) 209-219.

[8] H. Bateman, A. Erdelyi, Higher transcendental functions, 1953. 
[9] I.M. Gelfand, M.I. Graev, V.S. Retakh, General hypergeometric systems of equations and series of hypergeometric type, Russian Math. Surveys 47 (1992), no. 4, 1-88

[10] E.V. Ferapontov, A.V. Odesskii, Integrable Lagrangians and modular forms, arXiv:0707.3433.

[11] P.A. Burovskiy, E.V. Ferapontov and S.P. Tsarev, Second order quasilinear PDEs and conformal structures in projective space, arXiv:0802.2626 [nlin. SI].

[12] W. F. Ames, R. L. Anderson, V. A. Dorodnitsyn, E. V. Ferapontov, R. K. Gazizov, N. H. Ibragimov, S. R. Svirshchevskii, CRC handbook of Lie group analysis of differential equations. Vol. 1. Symmetries, exact solutions and conservation laws. CRC Press, Boca Raton, FL, 1994.

[13] E.V. Ferapontov, L. Hadjikos, K.R. Khusnutdinova, Integrable equations of the dispersionless Hirota type and hypersurfaces in the Lagrangian Grassmannian, arXiv:0705.1774.

[14] J. Gibbons, S.P. Tsarev, Reductions of Benney's equations, Phys. Lett. A, 211 (1996) 19-24. J. Gibbons, S.P. Tsarev, Conformal maps and reductions of the Benney equations, Phys. Lett. A, 258 (1999) 263-270.

[15] M. V. Pavlov, Algebro-geometric approach in the theory of integrable hydrodynamic-type systems. Comm. Math. Phys., 272(2) (2007) 469-505.

[16] A. A. Akhmetshin, I. M. Krichever, Y. S. Volvovski, A generating formula for solutions of associativity equations. Russian Math. Surveys 54 (1999), no. 2, 427-429.

[17] E.V. Ferapontov, K.R. Khusnutdinova, On integrability of (2+1)-dimensional quasilinear systems, Comm. Math. Phys. 248 (2004) 187-206, E. V. Ferapontov, K.R. Khusnutdinova, The characterization of 2-component (2+1)-dimensional integrable systems of hydrodynamic type, J. Phys. A: Math. Gen. 37(8) (2004) 2949-2963.

[18] E.V. Ferapontov, D.G. Marshal, Differential-geometric approach to the integrability of hydrodynamic chains: the Haanties tensor, Math. Ann. 339(1), (2007) 61-99.

[19] M. V. Pavlov, Classification of integrable hydrodynamic chains and generating functions of conservation laws, J. Phys. A: Math. Gen. 39(34) (2006) 10803-10819.

[20] E.V. Ferapontov, K.R. Khusnutdinova and S.P. Tsarev, On a class of three-dimensional integrable Lagrangians, Comm. Math. Phys. 261, no. 1 (2006) 225-243.

[21] S.P. Tsarev, On Poisson brackets and one-dimensional Hamiltonian systems of hydrodynamic type, Soviet Math. Dokl., 31 (1985) 488-491. S.P. Tsarev, The geometry of Hamiltonian systems of hydrodynamic type. The generalized hodograph method, Math. USSR Izvestiya, 37 No. 2 (1991) 397-419. 1048-1068. 
[22] M.V. Pavlov, S.P. Tsarev, Tri-Hamiltonian structures of the Egorov systems of hydrodynamic type. Func. Anal. and Appl. , 37(1) (2003) 32-45. 